\title{
Defect-trapped electrons and ferromagnetic exchange in $\mathrm{ZnO}$
}

\author{
Aurab Chakrabarty and Charles H. Patterson \\ School of Physics, Trinity College Dublin, Dublin 2, Ireland \\ (Received 29 January 2011; revised manuscript received 21 July 2011; published 11 August 2011)
}

\begin{abstract}
A model for ferromagnetism observed at ambient temperature in films of oxides such as $\mathrm{ZnO}$ is proposed and evaluated. The ferromagnetic moment in the model arises from electrons trapped at negatively charged vacancies in an $n$-type oxide. These vacancies are capable of trapping either one or two electrons. Trapped electrons are described by a one-band Hubbard Hamiltonian where the Hubbard $U$ is the effective electron-electron repulsion for a pair of electrons in a vacancy. Ferromagnetism is known to exist in the Hubbard model applied to periodic three-dimensional (3D) lattices, provided the Hubbard $U$ parameter exceeds the defect bandwidth $W$ and the filling is away from half or complete filling. Hybrid and local-density approximation density-functional theory calculations are used to evaluate Hubbard model parameters for electrons trapped in defects in $\mathrm{ZnO}$. They are also used to calculate magnetic exchange couplings of well-separated, singly negatively charged defects, which are induced by a conduction band electron. Strong ferromagnetic coupling between defects is found in these total-energy calculations over a range exceeding $10 \AA$ when the defects have a large, positive Hubbard $U$ value. Hubbard $U$ values for oxygen $\left(\mathrm{V}_{\mathrm{O}}\right)$, zinc $\left(\mathrm{V}_{\mathrm{Zn}}\right)$, and zinc-oxygen complex $\left(\mathrm{V}_{\mathrm{ZnO}}\right)$ vacancies in various charge states are estimated from defect transition levels. $\mathrm{V}_{\mathrm{ZnO}}^{-}$, the negatively charged $\mathrm{ZnO}$ pair vacancy, and $\mathrm{V}_{\mathrm{Zn}}^{-}$are proposed as possible sources of magnetic moment in ferromagnetic $\mathrm{ZnO}$ films. These vacancies can trap one or two electrons and their charge transition levels lie in the band gap. Some literature values of $U$ and those obtained here for unrelaxed vacancies are large enough to support a Hubbard model for ferromagnetism; however, $U$ values obtained depend strongly on lattice relaxation. The relaxed vacancies considered here have $U / W$ values which are not large enough for ferromagnetism using the simple criterion $U / W>1$.
\end{abstract}

DOI: 10.1103/PhysRevB.84.054441

PACS number(s): 71.55.Gs, 71.70.Gm, 75.30.Hx

\section{INTRODUCTION}

In the last decade, there have been numerous reports of room-temperature ferromagnetism in oxide thin films doped with transition metals (TM). ${ }^{1-8}$ More recently, there have been reports of room-temperature ferromagnetism in oxide thin films prepared without TM doping ${ }^{9-13}$ and the term " $d^{0}$ magnetism" has been coined. ${ }^{9}$ Many experimental studies invoke oxygen ${ }^{2,7,11,12,14-16}$ or metal cation defects ${ }^{9,10}$ in the exchange-coupling mechanism. Oxygen vacancies are postulated to be important because of a strong correlation between low oxygen partial pressures in film growth or processing and observation of ferromagnetism. Two X-ray magnetic circular dichroism (XMCD) studies ${ }^{6,7}$ of ferromagnetic $\mathrm{Zn}_{1-x} \mathrm{Co}_{x} \mathrm{O}$ thin films reported no evidence for ferromagnetic order in the $\mathrm{Co}^{2+}$ ions. A Mössbauer study of ferromagnetic Fe doped $\mathrm{TiO}_{2}$ found no ferromagnetic order of Fe ions. ${ }^{17}$ The primary sources of the aligned magnetic moments and mechanism for exchange coupling in these films therefore remain controversial. Together with reports of ferromagnetism in the undoped films just mentioned, it is possible that ferromagnetism in these films is intrinsic to films with vacancy defects.

Any theoretical model of ferromagnetism, which is applicable to a wide range of oxides ought to be generic: it should not depend on details of interactions in a particular system. This point has been emphasized recently. ${ }^{18,19}$ If vacancy defects are responsible for ferromagnetism in thin films, the likely requirements of a model for the exchange-coupling mechanism are the following: similar defects with intrinsic magnetic moments should exist in a range of oxides, they should be thermodynamically stable up to (at least) room temperature and the magnetic exchange coupling between them should be strong enough (at typical defect near-neighbor distances) to allow ferromagnetism to exist. One of the outstanding questions, which arises in this context, is how ferromagnetic exchange between paramagnetic vacancies is mediated over relatively long distances of order $10 \AA$.

Here, we outline and evaluate a model for ferromagnetism in oxide films with vacancy defects that is potentially universal. Vacancy defects that can trap one or two electrons are postulated to be the sites where magnetic moments are formed. The defects have an intrinsic magnetic moment when a single electron is trapped; when the population of these sites lies between one and two electrons per site, the ground state of the system is ferromagnetic, provided that the electron-electron interaction on the vacancy site is large enough. We report careful calculations of the Hubbard $U$ value for the $\mathrm{V}_{\mathrm{Zn}}$ and $\mathrm{V}_{\mathrm{ZnO}}$ vacancies, which are expected to be the most common vacancies that can trap one or two electrons in $n$-type $\mathrm{ZnO}$. In order to achieve a magnetization comparable to values observed in experiment, a defect density of order 1 at.\% is necessary.

Oxide films containing a reasonably high density of defects are modelled by a one-band Hubbard model. Ferromagnetism in one-band Hubbard models on lattices has been extensively studied by several techniques including the high-temperature series expansion (HTSE), ${ }^{20}$ the spectral density approach (SDA), ${ }^{21}$ dynamical mean-field theory (DMFT), ${ }^{22}$ and the quantum Monte Carlo (QMC) method. ${ }^{23}$ The dependence of the Curie temperature on filling factor is known, at least for ordered fcc, ${ }^{20,22} \mathrm{bcc},{ }^{20,21}$ and $\mathrm{sc}^{20,24}$ lattices; the ratio of Hubbard $U$ to bandwidth $W$, necessary for ferromagnetism to exist, depends on lattice type $\mathrm{e}^{21}$ and it can be as low as $\sim 1.5$ in the bcc lattice. ${ }^{21}$ We note that a Hubbard Hamiltonian may be an oversimplification of the Hamiltonian for electrons 
trapped at defect site. Nevertheless, it is likely that a large on-site repulsion is necessary for ferromagnetism mediated by electron exchange between defects with varying near-neighbor distances.

Hybrid density-functional-theory calculations (DFT) on $\mathrm{ZnO}$ supercells with $\mathrm{V}_{\mathrm{Zn}}, \mathrm{V}_{\mathrm{O}}$, or $\mathrm{V}_{\mathrm{ZnO}}$ vacancy defects are used to evaluate the viability of the model. The hybrid DFT method used here (see Appendix A for details) avoids the pitfalls of the local-density approximation (LDA) to DFT when applied to oxides, which have been highlighted recently. ${ }^{18}$ The hybrid DFT single-particle band gap predicted for $\mathrm{ZnO}$ is in reasonable agreement with experiment (3.26 cf. $3.47 \mathrm{eV}$ from Ref. 25), whereas it is grossly underestimated in LDA.

Hubbard $U$ parameters for trapped electron pairs are deduced from total-energy calculations on partialy filled vacancy electron traps in different spin states and from transition levels for $\mathrm{V}_{\mathrm{Zn}}$ and $\mathrm{V}_{\mathrm{ZnO}}$ in the 1- and 2- states. The $U / W$ ratio obtained this way depends strongly on whether the defect is allowed to relax to its equilibrium configuration in a particular charge state.

Current theoretical models for ferromagnetism in TMdoped oxide films include a spin-split donor-impurity band model, ${ }^{26,27}$ bound magnetic polarons, ${ }^{28,29}$ and direct exchange of $n$-type carriers between TM impurity spins. ${ }^{30}$ Our model is similar to a recent model proposed for ferromagnetism in $\mathrm{CaO}^{31}$ and a recent Stoner model ${ }^{17}$ for ferromagnetism in transition-metal impurity ions in $\mathrm{TiO}_{2}$.

The remainder of this paper is organized as follows: Sec. II gives an overview of experimental work on ferromagnetic oxide thin films, which is relevant to the exchange-coupling mechanism, Sec. III gives a short review of current theoretical models for ferromagnetism in TM-doped oxides, Sec. IV outlines the Hubbard model used in this work, Sec. V gives results of hybrid DFT calculations of defect formation energies, transition levels, and estimates of Hubbard $U$ and $t$ parameters as well as magnetic exchange coupling constant calculations for $\mathrm{Co}^{2+}$ near $\mathrm{V}_{\mathrm{ZnO}}^{-}$and $\mathrm{V}_{\mathrm{Zn}}^{-}$vacancies. Finally, these results are discussed and summarized in Secs. VI and VII.

\section{THIN FILM EXPERIMENTAL WORK}

In this section, we briefly review the experimental literature on ferromagnetism in transition-metal-doped $\mathrm{ZnO}$ and undoped metal oxide films. In particular, the importance of vacuum annealing of samples in the production of ferromagnetic films, the magnetic state of transition-metal ions in the films, and a correlation between magnetization and carrier density. Room-temperature ferromagnetism was initially reported in transition-metal-doped $\mathrm{ZnO}$ about a decade ago ${ }^{1}$ and thereafter by many research groups. ., $32-34$

A strong correlation has been established between vacuum annealing of samples and ferromagnetism in films. This has been reported in $\mathrm{Zn}_{1-x} \mathrm{Co}_{x} \mathrm{O}$, ${ }^{3,11,14-16,34,35} \mathrm{Zn}_{1-x} \mathrm{Mn}_{x} \mathrm{O},{ }^{36}$ $\mathrm{ZnO},{ }^{36} \mathrm{TiO}_{2},{ }^{12,13}$ and $\mathrm{HfO}_{2} \cdot{ }^{10}$ In one case, ${ }^{15}$ cycling between ferromagnetic and non-magnetic states by vacuum or air annealing was clearly demonstrated. However, in another study, ${ }^{10}$ annealing in $\mathrm{O}_{2}$ for 10 hours at $600^{\circ} \mathrm{C}$ did not destroy the ferromagnetism. The former observations have been widely used to argue that oxygen vacancies are important to ferromagnetism in both transition-metal-doped and undoped thin films. In an earlier paper, ${ }^{37}$ one of the authors of this work showed that $\mathrm{V}_{\mathrm{O}}$ was unlikely to be the defect responsible for ferromagnetism in $\mathrm{Zn}_{1-x} \mathrm{Co}_{x} \mathrm{O}$ since its only magnetic state $\mathrm{V}_{\mathrm{O}}^{+}$ was metastable. Furthermore, it does not exist in this charge state in $n$-type $\mathrm{ZnO}$ thin films. Yet the empirical observation of a strong correlation between film annealing in air or oxygen and appearance of ferromagnetism must be explained.

Two groups have reported $\mathrm{x}$-ray magnetic circular dichroism (XMCD) studies ${ }^{6,7,38}$ of $\mathrm{Zn}_{1-x} \mathrm{Co}_{x} \mathrm{O}$ in which $\mathrm{Co}$ ions in ferromagnetic films showed paramagnetic behavior, leading the authors to conclude that the Co ions were not the source of magnetization. Two visible-UV magnetic circular dichroism (MCD) studies of $\mathrm{Zn}_{1-x} \mathrm{M}_{x} \mathrm{O}$ with $M=\mathrm{Co}, \mathrm{Mn}, \mathrm{V}$, and Ti (see Refs. 4 and 5) showed a Faraday effect at $3 \mathrm{eV}$ in a region where the $\mathrm{ZnO}$ film was still transparent and that ferromagnetism in doped $\mathrm{ZnO}$ caused band splitting of the bulk $\mathrm{ZnO}$ electronic structure. It was also concluded that the $\mathrm{ZnO}$ conduction band was hybridized with the magnetic ions and was spin split. ${ }^{4}$ Paramagnetic ${ }^{39,40}$ and superparamagnetic ${ }^{39,41}$ transition-metal clusters and other ferromagnetic secondary phases ${ }^{42}$ have been identified in $\mathrm{ZnO}$. Ferromagnetism has also been reported in metal oxide thin films including $\mathrm{HfO}_{2},{ }^{9,43} \mathrm{TiO}_{2},{ }^{43,44} \mathrm{In}_{2} \mathrm{O}_{3},{ }^{43}$ $\mathrm{SnO}_{2},{ }^{45}$ and $\mathrm{ZnO} .{ }^{10,19}$ Since these oxides are nonmagnetic in their bulk form, vacancy defects may play an important role in ferromagnetism in these oxides.

The relationship between carrier density and magnetization was studied in $\mathrm{Zn}_{1-x} \mathrm{Co}_{x} \mathrm{O}$ films, which had been co-doped with $\mathrm{Al}^{5,46}$ in order to control the $n$-type carrier concentration. In one case, where the films were epitaxially grown, ${ }^{46}$ highly resistive films were obtained by film deposition in a high oxygen pressure: co-doping with $\mathrm{Co}$ and $\mathrm{Al}$ produced films of much lower resistivity but none of the films of high crystallinity showed any ferromagnetism. On the other hand, films produced under low oxygen pressure and co-doped with $\mathrm{Co}$ and $\mathrm{Al}$ showed a marked variation in magnetization with carrier density. ${ }^{5}$ Several films with $n$-type carrier densities below $3 \times 10^{18} \mathrm{~cm}^{-3}$ showed significant magnetization, there was an intermediate regime between that density and $1 \times$ $10^{20} \mathrm{~cm}^{-3}$ and films with carrier densities between that density and $1 \times 10^{21} \mathrm{~cm}^{-3}$ showed a magnetization which had a strong dependence on density, peaking around $6 \times 10^{20} \mathrm{~cm}^{-3}$.

The model, which we propose here, can account for strong carrier density dependence of magnetization through carrier-density-dependent filling of vacancy defect levels. Our model consists of single carriers trapped in vacancies, possibly either $\mathrm{V}_{\mathrm{Zn}}^{-}$or $\mathrm{V}_{\mathrm{ZnO}}^{-}$, with additional $n$-type carriers scattering from these trapped electrons. It maps onto a one-band Hubbard model, which has been demonstrated to have strong magnetization dependence on carrier density. Hence, according to the model, one would expect to find carrier-density-dependent ferromagnetism in samples with defects, but not in $\mathrm{Co}$ and $\mathrm{Al}$ co-doped samples with high crystalline quality.

\section{CURRENT THEORETICAL MODELS}

Theoretical models for ferromagnetism in $\mathrm{ZnO}$ and other oxides can be divided into models which assume direct ferromagnetic coupling of transition-metal moments by carrier electrons and models in which $\mathrm{V}_{\mathrm{O}}$ or $\mathrm{V}_{\mathrm{Zn}}$ vacancies 
play a role. Direct coupling of transition-metal magnetic moments by conduction electrons has been proposed by several groups ${ }^{30,47-49}$ using DFT, ${ }^{47,48}$ DFT + Hubbard $U,{ }^{30,49}$ or hybrid $\mathrm{DFT}^{48}$ methods. These calculations used relatively small supercells $\left(2 \times 2 \times 1,{ }^{47} 2 \times 2 \times 2,{ }^{48}\right.$ or $\left.3 \times 3 \times 2^{30,49}\right)$ so that electron concentrations are very high (ranging from $2 \times 10^{21}$ to $9 \times 10^{21} \mathrm{~cm}^{-3}$ at one electron per supercell). These studies find that once the Fermi level reaches the vacant, minority-spin $d$ states, the ferromagnetic state is strongly favored energetically. Models in which exchange coupling of TM ions is mediated by conduction band electrons alone obviously cannot be applied to undoped oxides. These models are also in disagreement with the experimental observation that no ferromagnetism is reported in highly crystalline $\mathrm{Zn}_{1-x} \mathrm{Co}_{x} \mathrm{O}$ films, which are co-doped with $\mathrm{Al} .{ }^{46}$

Several studies have considered the possible role of $\mathrm{V}_{\mathrm{O}}$ in promoting ferromagnetism. ${ }^{27,37,50}$ A DFT study on $\mathrm{Zn}_{1-x} \mathrm{Co}_{x} \mathrm{O}$ and $\mathrm{Zn}_{1-x} \mathrm{Mn}_{x} \mathrm{O}^{50}$ found that $\mathrm{V}_{\mathrm{O}}$ had little effect on magnetic couplings of the transition-metal ions. A hybrid DFT study by one of the authors ${ }^{37}$ found that $\mathrm{V}_{\mathrm{O}}$ in the $1+$ state could induce some ferromagnetic coupling between $\mathrm{Co}^{2+}$ ions in $\mathrm{Zn}_{1-x} \mathrm{Co}_{x} \mathrm{O}$, but the $\mathrm{V}_{\mathrm{O}}^{+}$vacancy was metastable. A self-interaction corrected (SIC) DFT study ${ }^{27}$ concluded that ferromagnetism in $\mathrm{Zn}_{1-x} \mathrm{Co}_{x} \mathrm{O}$ originates from $\mathrm{Co}_{\mathrm{Zn}} / \mathrm{V}_{\mathrm{O}}$ pairs with a partially filled level close to the conduction band. The coupling mechanism in this case is somewhat similar to direct coupling by conduction band electrons in that a hybridized transition metal $d$ and $\mathrm{V}_{\mathrm{O}}$ state becomes occupied in the ferromagnetic state. This model is similar to the impurity band exchange model ${ }^{26}$ in which a shallow spin-split donor band is associated with a polarized electron localized at oxygen vacancy sites. Two recent DFT studies have proposed that the $\mathrm{V}_{\mathrm{Zn}}$ vacancy is the defect responsible for ferromagnetism in $\mathrm{ZnO}$ films. ${ }^{51,52}$

\section{HUBBARD MODEL}

In the model considered here, vacancy defects trap one electron in a state with a magnetic moment of $1 \mu_{B}$ and additional electron carriers are scattered by these trapped electrons. The number of electrons per vacancy defect lies between one and two. Hybrid DFT calculations show that when a single electron is trapped, the trapped electron wave function is largely confined to the defect. However, when a second electron is added to a supercell containing one $\mathrm{V}_{\mathrm{ZnO}}$ vacancy defect, the trapped electrons' wave function is more extended and may be a resonance in which the localized defect wave function mixes with the bottom of the conduction band. This leads to a large hopping amplitude between defects when there is more than one electron per defect and a greater defect level bandwidth.

The essential physics of magnetism in undoped oxide thin films in this model is projected onto a single-band Hubbard model:

$$
H=t_{i j} c_{i \sigma}^{\dagger} c_{j \sigma}+\frac{1}{2} U n_{i \sigma} n_{i-\sigma},
$$

in which a defect is represented as a site, $U$ is the on-site coulomb repulsion energy, and $t$ is a hopping parameter. In an oxide with defects which are not ordered, the hopping parameter will depend on defect separation but the Hubbard
$U$ value is not expected to change significantly for a particular defect type. There are threshold carrier densities and Hubbard $U$ parameters, which are necessary for ferromagnetism in simple, ordered lattices. Nielsen and Bhatt ${ }^{53}$ have considered a similar model to ours for ferromagnetism in finite systems without transition-metal ions. They used a Hubbard model on finite lattices and disordered clusters in which the hopping parameter depended on the occupancies of both sites involved in a hop and on the site separation. Hubbard parameters were obtained from first-principles calculations on interstitial $\mathrm{H}^{-}$ ions, which have a much larger $U$ value than the defects considered here, owing to the greater confinement of the trapped electron. They found similar magnetization for both (ordered) finite lattices and disordered clusters.

Ferromagnetism in the phase diagram for the Hubbard model on ordered lattices at finite temperatures ${ }^{21-23,54}$ requires the ratio $U / W$ to be greater than unity, ${ }^{54}$ depends on the single-particle density of states ${ }^{23}$ and exists only away from half-filling of the sites. $W$ is the single-particle bandwidth and half-filling corresponds to one electron per site. The magnetization versus site-filling phase diagram is symmetric about half filling for bipartite lattices (bcc, sc, etc.) with particle-hole symmetry. Otherwise (e.g., fcc), ferromagnetism exists only above half filling. In the large $U$ limit, the Curie temperatures for sc, bcc, and fcc lattices lie in the range 0.8 to $1.8 t$, according to HTSE calculations by Henderson et al. ${ }^{20}$ We find values of $t(70 \mathrm{meV}), W(400 \mathrm{meV})$, and $U$ in excess of $1 \mathrm{eV}$ for unrelaxed $\mathrm{V}_{\mathrm{ZnO}}$ vacancies separated by just over $11 \AA$. These values place this system in the large $U$ limit and corresponding Curie temperatures predicted by HTSE calculations ${ }^{20}$ lie in the range 650 to $1460 \mathrm{~K}$. However, the value of $U$ decreases significantly when the $\mathrm{V}_{\mathrm{ZnO}}$ vacancy is allowed to relax.

\section{RESULTS}

\section{A. Intrinsic magnetic defects in $\mathrm{ZnO}$}

$\mathrm{V}_{\mathrm{O}}^{+}$is a spin-1/2 magnetic state of the oxygen vacancy. It is a negative $U$ defect and is metastable with respect to dissociation into $\mathrm{V}_{\mathrm{O}}$ and $\mathrm{V}_{\mathrm{O}}^{2+}$ (see Refs. 37 and 55-57) and is, therefore, unlikely to be found in $n$-type $\mathrm{ZnO}$. $\mathrm{V}_{\mathrm{O}}$ does not trap an electron to form $\mathrm{V}_{\mathrm{O}}^{-}$according to hybrid DFT supercell calculations; an electron added to a supercell containing $\mathrm{V}_{\mathrm{O}}$ is accommodated in the conduction band and not in the vacancy.

The $\mathrm{V}_{\mathrm{Zn}}$ vacancy is a deep acceptor with transition levels $\epsilon(0 /-)$ and $\epsilon(-/ 2-)$ reported to be $E_{V}+0.1$ (Ref. 58) and $E_{V}+1.0 \mathrm{eV}$ (Ref. 59), according to theory and experiment, respectively; below we report a value of $E_{V}+0.64 \mathrm{eV}$ for both transition levels of $\mathrm{V}_{\mathrm{Zn}}$. Energy $E_{V}$ is the valence band maximum energy. It has a triplet spin-1 ground state. ${ }^{60}$ Vacancy defects identified as $\mathrm{V}_{\mathrm{Zn}}$ by positron annihilation spectroscopy (PAS) were removed by annealing above $600{ }^{\circ} \mathrm{C} .{ }^{61}$ The neutral spin-1 $\mathrm{V}_{\mathrm{Zn}}$ vacancy reported by Galland and Hervé in $1970^{62}$ and more recently ${ }^{63,64}$ shows the two lines characteristic of two holes in a triplet state.

Neutral $\mathrm{ZnO}$ pair vacancies $\mathrm{V}_{\mathrm{ZnO}}$, in which $\mathrm{V}_{\mathrm{O}}$ and $\mathrm{V}_{\mathrm{Zn}}$ vacancies are separated by a single $\mathrm{Zn}-\mathrm{O}$ bond distance, have no occupied dangling electron or unoccupied hole states. In this work, we consider only $\mathrm{V}_{\mathrm{ZnO}}$ pair vacancies where the vacancy 


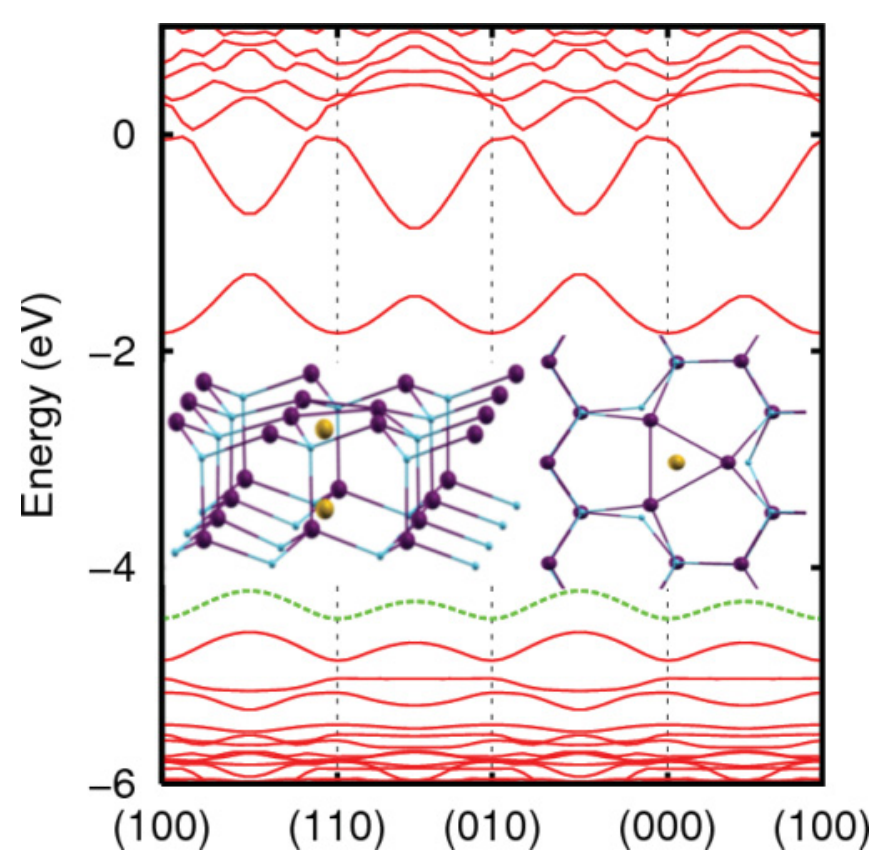

FIG. 1. (Color online) Electronic band structure for a $\mathrm{V}_{\mathrm{ZnO}}^{2-}$ cluster vacancy in a $2 \sqrt{ } 3 \times 2 \sqrt{ } 3 \times 2$ supercell (solid red lines). The position of the occupied electronic state containing the trapped electron in singly-charged $\mathrm{V}_{\mathrm{ZnO}}^{-}$is superimposed (dotted green line). This band shifts upward by over $2 \mathrm{eV}$ when this state becomes doubly occupied. Inset shows two views of the crystal structure surrounding the $\mathrm{V}_{\mathrm{ZnO}}^{2-}$ cluster vacancy: $\mathrm{Zn}$ ions (large, dark spheres), O ions (small, light spheres), vacant $\mathrm{Zn}$ and $\mathrm{O}$ ion sites (large, light spheres). The $\mathrm{Zn}-\mathrm{Zn}$ distance for $\mathrm{Zn}$ ions, which are nearest neighbors of the vacancy $\mathrm{O}$ site, is reduced to $2.62 \AA$.

axis is aligned with the crystallographic $c$ axis. $\mathrm{V}_{\mathrm{ZnO}}$ can bind one or two electrons to form $\mathrm{V}_{\mathrm{ZnO}}^{-}$or $\mathrm{V}_{\mathrm{ZnO}}^{2-}$. The structure of the $\mathrm{V}_{\mathrm{ZnO}}^{2-}$ defect, obtained by relaxing the positions of all ions in a $2 \sqrt{ } 3 \times 2 \sqrt{ } 3 \times 2$ supercell containing 94 ions, is shown in Fig. 1, along with the electronic band structure for the defect. Four dangling $\mathrm{O} 2 p$ states surrounding a $\mathrm{V}_{\mathrm{Zn}}$ vacancy form nondegenerate $a$ and triply-degenerate $t$ band orbitals at the $\Gamma$ point of the Brillouin zone. The electronic configuration of neutral $\mathrm{V}_{\mathrm{Zn}}$ in wurtzite $\mathrm{ZnO}$ is $a^{2} t^{2} t^{1} t^{1}$, i.e., the $t$ band orbital has one doubly occupied and two singly occupied states, which form a spin triplet (see Fig. 5 in Ref. 37). $V_{O}$ has two dangling electrons; when $\mathrm{V}_{\mathrm{O}}$ and $\mathrm{V}_{\mathrm{Zn}}$ combine, these dangling electrons completely fill the $t$ states, leaving no dangling electrons in the neutral $\mathrm{V}_{\mathrm{ZnO}}$ vacancy.

Three doubly occupied $\mathrm{O} 2 p$ states in $\mathrm{V}_{\mathrm{ZnO}}$ occur between 0.2 and $1.0 \mathrm{eV}$ above the bulk valence band maximum (VBM) (around $-5 \mathrm{eV}$ in Fig. 1). Dangling $\mathrm{Zn} 4 s$ states surrounding a $\mathrm{V}_{\mathrm{O}}$ vacancy form an $a$ band orbital at the $\Gamma$ point of the Brillouin zone (see Fig. 4 in Ref. 37). This band is doubly occupied in neutral $\mathrm{V}_{\mathrm{O}}$. In neutral $\mathrm{V}_{\mathrm{ZnO}}$, this band is empty, in $\mathrm{V}_{\mathrm{ZnO}}^{-}$, it is singly occupied and a large splitting of the occupied and empty single-particle levels is found. In $\mathrm{V}_{\mathrm{ZnO}}^{2-}$, it is doubly occupied and the occupied level in $\mathrm{V}_{\mathrm{ZnO}}^{-}$shifts upward by around $2.7 \mathrm{eV}$, indicating a large electronic $U$ value for the state. The positions of this level (doubly occupied in $\mathrm{V}_{\mathrm{ZnO}}^{2-}$ and singly occupied in $\mathrm{V}_{\mathrm{ZnO}}^{-}$) are shown as a solid red line around $-1.6 \mathrm{eV}$ and a dotted green line around $-4.3 \mathrm{eV}$ in Fig. 1.

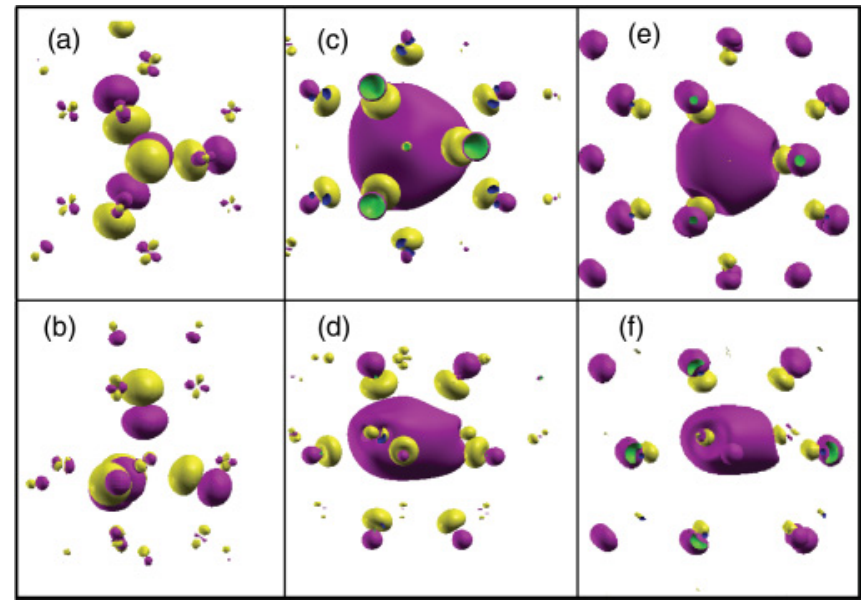

FIG. 2. (Color online) Wave functions for trapped electrons in $\mathrm{V}_{\mathrm{Zn}}^{-}, \mathrm{V}_{\mathrm{ZnO}}^{-}$, and $\mathrm{V}_{\mathrm{ZnO}}^{2-}$ vacancies at the $\Gamma$ point of the Brillouin zone. Trapped electron wave function in $\mathrm{V}_{\mathrm{Zn}}^{-}$(a) viewed along the $c$ axis, (b) viewed perpendicular to the $c$ axis. Trapped electron wave function in $\mathrm{V}_{\mathrm{ZnO}}^{-}$(c) viewed along the $c$ axis, (d) viewed perpendicular to the $c$ axis. Trapped electron wave function in $\mathrm{V}_{\mathrm{ZnO}}^{2-}$ (e) viewed along the $c$ axis, (f) viewed perpendicular to the $c$ axis.

$\mathrm{V}_{\mathrm{ZnO}}^{-}$has a magnetic moment of $1 \mu_{B}$. Wave functions for one or two electrons trapped in $\mathrm{V}_{\mathrm{ZnO}}$ vacancies and one electron in $\mathrm{V}_{\mathrm{Zn}}^{-}$are shown in Fig. 2. The overall character of the wave function when two electrons are trapped in $\mathrm{V}_{\mathrm{ZnO}}^{2-}$ is similar, but the extent to which the wave function for the trapped electron pair spreads into the bulk via the conduction band is larger than for the single trapped electron. Unlike $\mathrm{V}_{\mathrm{ZnO}}^{2-}$, when $\mathrm{V}_{\mathrm{Zn}}^{-}$is transformed into $\mathrm{V}_{\mathrm{Zn}}^{2-}$, there is no large upward shift of the $t$ states, which are being occupied, indicating a small electronic Hubbard $U$ value. The relaxed crystal structure for $\mathrm{V}_{\mathrm{ZnO}}^{2-}$ in Fig. 1 shows that the trapped electron pair mainly resides in a $\mathrm{Zn}_{3}$ "colloid" where the $\mathrm{Zn}-\mathrm{Zn}$ distance is reduced from $3.25 \AA$ in bulk $\mathrm{ZnO}$ to $2.62 \AA$, close to the nearest-neighbor distance in $\mathrm{Zn}$ metal $(2.66 \AA)$.

\section{B. Defect formation energies}

The formation energy for a defect $\mathrm{X}$ with charge $q, \mathrm{E}_{X^{q}}^{f}$, is 55,65

$$
E_{X^{q}}^{f}=E_{X^{q}}-E_{\text {bulk }^{0}}+n_{i} \mu_{i}+q\left(E_{F}-E_{V}\right),
$$

where $E_{\mathrm{bulk}^{0}}$ and $E_{X^{q}}$ are the total energies from supercell calculations for the bulk crystal and the bulk crystal with a defect $\mathrm{X}$ in charge state $q$, respectively. Variable $\mathrm{n}_{i}$ is the change in number of species $i$, removed from or added to the supercell to create the defect, $\mu_{i}$ is the chemical potential of species $i$, and $E_{F}-E_{V}$ is the position of the Fermi level relative to the valence band maximum level. Appropriate values for the chemical potentials of the crystal constituent elements have been discussed in Refs. 65 and 58 and elsewhere. The formalism used to calculate formation energies assumes that the chemical potential of $\mathrm{Zn}$ is equal to that of $\mathrm{Zn}$ in the solid state. The chemical potential for $\mathrm{O}$ relative to the standard chemical potential of $\mathrm{O}, \mu_{\mathrm{O}}^{\ominus}$, in the oxygen molecule is

$$
\mu_{\mathrm{O}}=\mu_{\mathrm{O}}^{\ominus}+k T \ln \frac{P}{P^{\ominus}},
$$


where $P$ is the oxygen partial pressure and ideal gas behavior has been assumed. Under typical oxide thin film growth conditions, the oxygen pressure in the growth chamber is in the range $10^{-2}$ to $10^{-1} \mathrm{mBar}^{3,46}$ At a typical film surface temperature of $500{ }^{\circ} \mathrm{C}$, the oxygen atom chemical potential is -0.6 to -0.8 $\mathrm{eV}$ less than at standard temperature and pressure. In previous defect formation energy calculations, ${ }^{58,65}$ the oxygen chemical potential $\mu_{\mathrm{O}}$ was assumed to lie in the range

$$
\mu_{\mathrm{O}}^{\ominus}\left[O_{2}\right]>\mu_{\mathrm{O}}>\mu_{\mathrm{O}}^{\ominus}\left[\mathrm{O}_{2}\right]+\Delta H^{f}[\mathrm{ZnO}],
$$

where the experimental value of $\Delta H^{f}(\mathrm{ZnO})$ is $-3.5 \mathrm{eV},{ }^{66}$ and to take the value, $\mu_{\mathrm{O}}^{\ominus}\left[\mathrm{O}_{2}\right]+\Delta H^{f}[\mathrm{ZnO}]$, under oxygen-poor conditions. In this work the chemical potentials, $\mu_{\mathrm{O}}$ and $\mu_{\mathrm{Zn}}$, and heat of formation of $\mathrm{ZnO}$ are approximated using the total energies of the bulk $\mathrm{ZnO}$ crystal, the $\mathrm{O}_{2}$ molecule in its triplet ground state and bulk $\mathrm{Zn}$ metal. Values for $\Delta H^{f}[\mathrm{ZnO}]$ of -4.5 and $-3.9 \mathrm{eV}$ are obtained from B3LYP and LDA calculations using the CRYSTAL code ${ }^{67}$ and compare to an experimental value of $-3.5 \mathrm{eV}$ (see Ref. 66) and $-3.6 \mathrm{eV}$ from another LDA calculation. ${ }^{58}$

Oxide thin films of interest in this work are typically grown with low oxygen partial pressures and so the chemical potential for oxygen, $\mu_{\mathrm{O}}^{\ominus}\left[\mathrm{O}_{2}\right]+\Delta H^{f}[\mathrm{ZnO}]$, is used. As just noted, this value lies below the actual oxygen chemical potential, but facilitates comparison of defect formation energies with those already in the literature. It also results in predictions of negative formation energies for the $\mathrm{V}_{\mathrm{O}}$ vacancy at low Fermilevel positions (see below).

The total energy of a periodic system containing a charged defect includes contributions from interactions between the defect charge and its images in other unit cells and a uniform compensating charge, ${ }^{68}$ added to make the unit cell charge neutral overall. Methods used to obtain defect formation energies and transition levels in this work are described in Appendix B. Formation energies from this work reported in Table I were obtained for fully relaxed $3 \times 3 \times 3$ supercells. They have not been corrected for defect-defect coulombic interactions. For defect concentrations relevant to the model for oxide ferromagnetism investigated here, a defect density of around one percent is expected and so defect-defect interactions will be present in the system being modeled.

Calculations in this work were performed using the Dirac exchange approximation ${ }^{69}$ and the Vosko, Wilks, and Nusair approximation to correlation ${ }^{70}$ in the LDA and B3LYP ${ }^{71,72}$ hybrid DFT Hamiltonians (see Appendix A). In earlier work, ${ }^{58}$ various schemes were applied to formation energies and transition levels calculated using an LDA Hamiltonian, in order to correct raw values obtained for the LDA band-gap error. No correction is made to formation energies derived from LDA or hybrid DFT calculations in this work to facilitate a direct comparison of the predictions of the two methods; as noted above, the hybrid DFT method used here predicts a bulk $\mathrm{ZnO}$ band gap in reasonable agreement with experiment, $3.26 \mathrm{eV}$ compared to $3.47 \mathrm{eV},{ }^{25}$ and no band-gap correction is needed.

Single-vacancy defect formation energies for oxygen-poor conditions, with the Fermi energy at the valence band maximum, are given in Table I along with selected literature values. Variation of defect formation energy with Fermi-level position is derived from values in Table I and Eq. (2). Formation
TABLE I. Formation energies in electron volts of single vacancy defects under oxygen-poor conditions with $E_{F}=E_{V}$.

\begin{tabular}{lcccccc}
\hline & $\mathrm{V}_{\mathrm{O}}$ & $\mathrm{V}_{\mathrm{O}}^{+}$ & $\mathrm{V}_{\mathrm{O}}^{2+}$ & $\mathrm{V}_{\mathrm{Zn}}$ & $\mathrm{V}_{\mathrm{Zn}}^{-}$ & $\mathrm{V}_{\mathrm{Zn}}^{2-}$ \\
\hline B3LYP $^{\mathrm{a}}$ & 0.5 & -1.2 & -3.7 & 7.5 & 8.1 & 8.7 \\
$\mathrm{HSE}^{\mathrm{b}}$ & 1.0 & $\ldots$ & -3.4 & 7.1 & 8.0 & 10.1 \\
$\mathrm{GGA}^{\mathrm{c}}$ & 1.0 & 0.3 & -0.5 & 5.4 & $\ldots$ & $\ldots$ \\
$\mathrm{LDA}^{\mathrm{a}}$ & 0.4 & $\ldots$ & $\ldots$ & 5.1 & $\ldots$ & $\ldots$ \\
$\mathrm{LDA}^{\mathrm{d}}$ & 0.0 & 0.2 & -0.3 & 5.5 & 5.8 & 6.6 \\
$\mathrm{LDA}^{\mathrm{e}}$ & 1.5 & 0.8 & -0.5 & 5.8 & 5.7 & 5.8 \\
$\mathrm{LDA}^{\mathrm{f}}$ & 0.7 & 0.6 & -0.4 & 5.9 & 6.0 & 6.3 \\
$\mathrm{LDA}^{+}+U^{\mathrm{f}}$ & 1.34 & 0.81 & -0.6 & 6.4 & 6.5 & 6.9 \\
$\mathrm{GGA}+U^{\mathrm{c}}$ & 1.71 & 0.71 & -0.7 & 5.6 & 6.0 & 7.1 \\
\hline \hline
\end{tabular}

aThis work.

${ }^{b}$ Heyd, Scuseria, and Ernzerhof hybrid density functional, ${ }^{73,74}$ Ref. 75.

${ }^{\mathrm{c}}$ Ref. 76.

${ }^{\mathrm{d}}$ Ref. 55.

${ }^{\mathrm{e}}$ Ref. 56.

${ }^{\mathrm{f}}$ Ref. 58 .

energies of $\mathrm{V}_{\mathrm{O}}, \mathrm{V}_{\mathrm{Zn}}$, and $\mathrm{V}_{\mathrm{ZnO}}$ in relevant charge states are shown in Fig. 3 as a function of Fermi-level position. Formation energies denoted "relaxed" in Fig. 3 were calculated using ion positions, which were relaxed for each charge state of the defect; alternatively, formation energies denoted "unrelaxed" were calculated using ion positions where all ions (except those removed to create the vacancy) were in their equilibrium bulk positions. The data in Fig. 3 show that predicted charge states of vacancies as a function of Fermi-level position depend strongly on atom relaxation. In particular, without relaxation all vacancies considered are predicted to be positive $U$ defects, so that singly charged states of vacancies are observed over wide Fermi-level ranges, while after relaxation, both $\mathrm{V}_{\mathrm{O}}$ and $\mathrm{V}_{\mathrm{ZnO}}$ vacancies become negative $U$ defects and the $U$ value for $\mathrm{V}_{\mathrm{Zn}}$ becomes zero.

According to literature values in Table I, the formation energy for $\mathrm{V}_{\mathrm{O}}$ ranges from 0.02 to $1.5 \mathrm{eV}$ in early LDA calculations (Refs. 55 and 56) or $1.71 \mathrm{eV}$ in a generalized gradient approximation plus $U(\mathrm{GGA}+U)$ calculation; ${ }^{76}$ more recent LDA, GGA, and hybrid DFT calculations place the formation energy for neutral $\mathrm{V}_{\mathrm{O}}$ in the range 0.4 to $1.0 \mathrm{eV}$, under oxygen poor conditions. The values, which we obtain from LDA and B3LYP calculations, are 0.4 and $0.5 \mathrm{eV}$, respectively. There is good agreement between LDA and B3LYP calculations of the $\mathrm{V}_{\mathrm{O}}$ formation energy in this work, but these methods predict quite different defect formation energies for charged states of defects, and therefore, different transition levels.

The $\mathrm{V}_{\mathrm{O}}^{+}$vacancy has been shown previously to be a metastable state of the oxygen vacancy. Calculated values for its formation energy, with the Fermi energy at the VBM level, range from $-1.2 \mathrm{eV}$ using the B3LYP hybrid functional to $0.81 \mathrm{eV}$ using $\mathrm{LDA}+U .{ }^{58}$ The formation energy for $\mathrm{V}_{\mathrm{O}}^{2+}$ is predicted by the HSE and B3LYP hybrid functionals to be -3.4 and $-3.7 \mathrm{eV}$, respectively, while $\mathrm{LDA}$ or $\mathrm{LDA}+U$ methods place the formation energy of $\mathrm{V}_{\mathrm{O}}^{2+}$ in the range -0.3 to $-0.7 \mathrm{eV}$. Underestimation of the gas phase chemical potential 

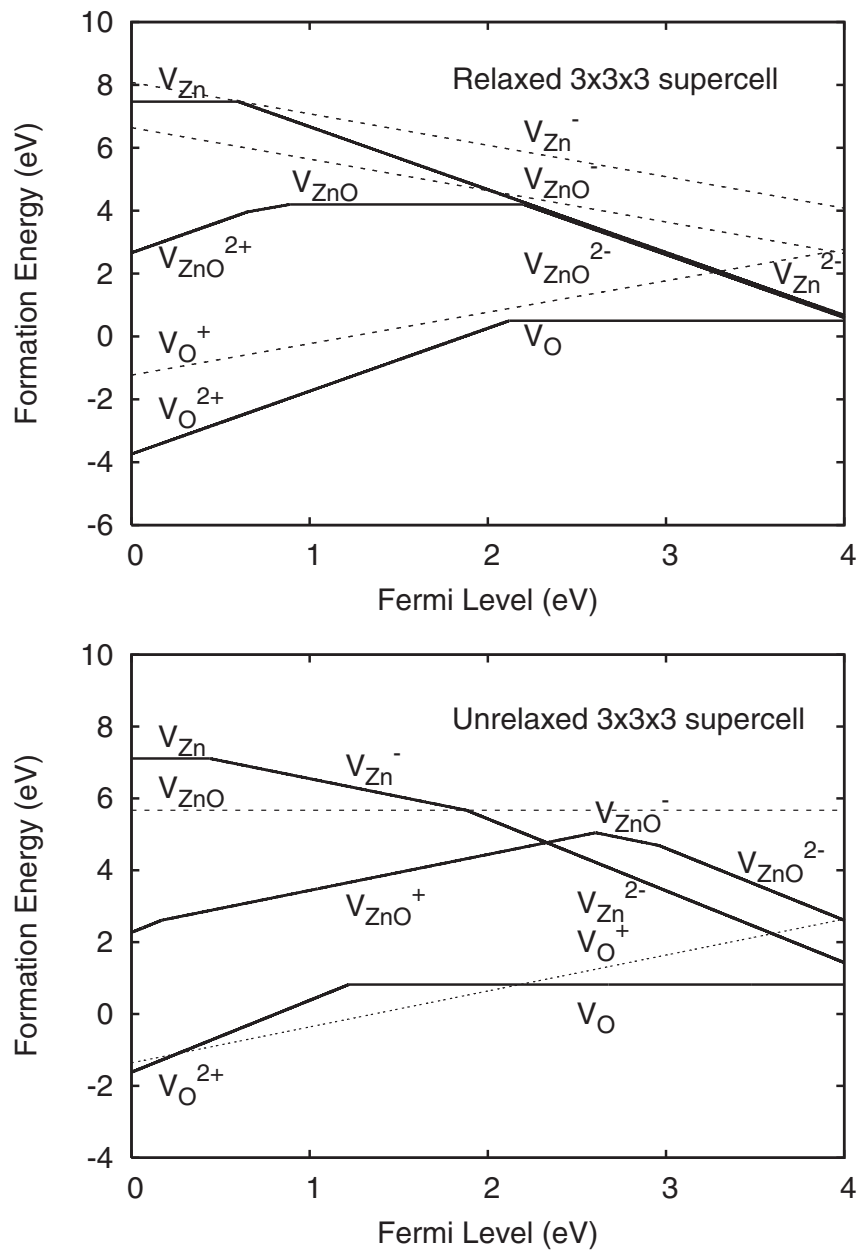

FIG. 3. Defect formation energies as a function of Fermi-level position for $\mathrm{V}_{\mathrm{O}}, \mathrm{V}_{\mathrm{Zn}}$, and $\mathrm{V}_{\mathrm{ZnO}}$ obtained using a B3LYP hybrid DFT Hamiltonian. (Upper panel) Formation energies obtained using fully relaxed $3 \times 3 \times 3$ supercells. (Lower panel) Formation energies obtained using unrelaxed $3 \times 3 \times 3$ supercells.

for oxygen by around $3 \mathrm{eV}$, as noted above, results in a large negative formation energy for $\mathrm{V}_{\mathrm{O}}^{2+}$.

Values for the formation energy of the neutral $\mathrm{V}_{\mathrm{Zn}}$ vacancy, under oxygen-poor/zinc-rich conditions, range from 5.1 to $7.5 \mathrm{eV}$ (Table I). Modifying the Hamiltonian from LDA or GGA to $\mathrm{LDA}+U$ or $\mathrm{GGA}+U$, or by using a hybrid functional rather than LDA, increases the formation energy for $\mathrm{V}_{\mathrm{Zn}}$ by up to $2.4 \mathrm{eV}$. Figure 3 shows that the charge states with the lowest formation energy for relaxed $V_{Z n}$ are $V_{Z n}$ and $\mathrm{V}_{\mathrm{Zn}}^{2-}$, for Fermi energies below or above the $0.6 \mathrm{eV} \epsilon(2-/ 0)$ transition level. This does not mean that these calculations predict that $\mathrm{V}_{\mathrm{Zn}}^{-}$is not observed. The predicted energy of $\mathrm{V}_{\mathrm{Zn}}$ plus $\mathrm{V}_{\mathrm{Zn}}^{2-}$ is the same as twice the energy of $\mathrm{V}_{\mathrm{Zn}}^{-}$, so that when electrons are added to a $\mathrm{ZnO}$ sample containing uncharged $\mathrm{V}_{\mathrm{Zn}}$ vacancies, the Fermi level will shift to $+0.6 \mathrm{eV}$ and a mixture of the three charge states will be observed.

Formation energies for $\mathrm{V}_{\mathrm{ZnO}}$ in its various charge states are given in Table II and combined defect formation energies for separate $V_{O}$ and $V_{Z n}$ vacancies are compared to formation energies for the $\mathrm{V}_{\mathrm{ZnO}}$ pair vacancy for various net charge states as a function of Fermi-level position in Fig. 4. Formation of
TABLE II. Formation energies in electron volts of $\mathrm{V}_{\mathrm{ZnO}}$ in various charge states under oxygen-poor conditions with $E_{F}=E_{V}$.

\begin{tabular}{lccccc}
\hline \hline & $\mathrm{V}_{\mathrm{ZnO}}^{2+}$ & $\mathrm{V}_{\mathrm{ZnO}}^{+}$ & $\mathrm{V}_{\mathrm{ZnO}}^{0}$ & $\mathrm{~V}_{\mathrm{ZnO}}^{-}$ & $\mathrm{V}_{\mathrm{ZnO}}^{2-}$ \\
\hline B3LYP $^{\mathrm{a}}$ & 2.7 & 3.3 & 4.2 & 6.6 & 8.4 \\
LDA $^{\mathrm{a}}$ & $\cdots$ & $\cdots$ & 4.0 & $\cdots$ & $\cdots$ \\
\hline \hline
\end{tabular}

This work.

$\mathrm{V}_{\mathrm{ZnO}}$ or $\mathrm{V}_{\mathrm{ZnO}}^{2-}$ is favored over separated vacancies by around $1 \mathrm{eV}$ for all Fermi-level positions, except for a narrow range of Fermi-level positions where $\mathrm{V}_{\mathrm{ZnO}}$ is positively charged. This vacancy was also considered by Vidya et al. in a recent DFT study of cluster vacancies in $\mathrm{ZnO} .{ }^{77}$ They found that the $\mathrm{V}_{\mathrm{ZnO}}$ vacancy is bound with respect to the separated vacancies by just over $1 \mathrm{eV}$, in all charge states. The results reported in Fig. 4 were obtained from formation energies of single-vacancy defects in $3 \times 3 \times 3$ supercells. The large binding energy of the $\mathrm{V}_{\mathrm{ZnO}}$ defect was confirmed by comparing the total energies of $\mathrm{V}_{\mathrm{Zn}}^{-}$and $\mathrm{V}_{\mathrm{O}}$ defects in a $3 \times 3 \times 3$ supercell with that from a $\mathrm{V}_{\mathrm{ZnO}}^{-}$defect in a $3 \times 3 \times 3$ supercell. The binding energy of $\mathrm{V}_{\mathrm{ZnO}}^{-}$obtained from single-vacancy formation energies was 1.9(4) $\mathrm{eV}$, which compares to $2.0(1) \mathrm{eV}$ from total energy calculations. $\mathrm{V}_{\mathrm{O}}$ and $\mathrm{V}_{\mathrm{Zn}}$ annealing temperatures are predicted to be $909 \mathrm{~K}$ for $\mathrm{V}_{\mathrm{O}}, 655 \mathrm{~K}$ for $\mathrm{V}_{\mathrm{O}}^{2+}$, and $539 \mathrm{~K}$ for $\mathrm{V}_{\mathrm{Zn}}^{2-}$ (Ref. 58) and, hence, at least $\mathrm{Zn}$ vacancies are expected to be mobile under oxide thin film growth conditions. Under oxygen-poor conditions and in the presence of $n$-type carriers, many $\mathrm{O}$ vacancies are therefore expected to exist as $\mathrm{V}_{\mathrm{ZnO}}$ pair vacancies (see Fig. 4).

\section{Defect transition levels}

Transition levels for charged defects are obtained by comparing formation energies of a defect in two distinct charge states ( $q$ and $q^{\prime}$, say) and solving for the Fermi-level position at which they become equal, $\epsilon\left(q / q^{\prime}\right)$, which is

$$
\epsilon\left(q / q^{\prime}\right)=E_{v}+\frac{E_{\mathrm{tot}}\left(X^{q^{\prime}}\right)-E_{\mathrm{tot}}\left(X^{q}\right)}{q-q^{\prime}} .
$$

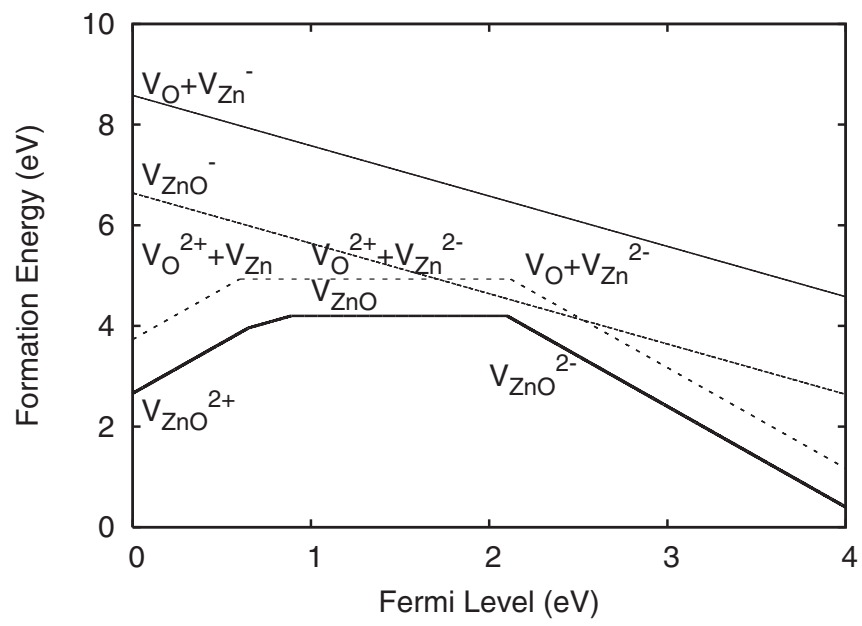

FIG. 4. Combined defect formation energies as a function of Fermi-level position for $\mathrm{V}_{\mathrm{O}}$ and $\mathrm{V}_{\mathrm{Zn}}$ compared to the formation energy for $\mathrm{V}_{\mathrm{ZnO}}$ obtained using a B3LYP Hamiltonian. 
TABLE III. Transition levels in electron volts relative to $E_{V}$.

\begin{tabular}{|c|c|c|c|c|c|c|}
\hline Defect & $q / q^{\prime}$ & $\mathrm{LDA}^{\mathrm{a}}$ & $\mathrm{LDA}+U^{\mathrm{a}}$ & B3LYP & B3 $\mathrm{LYP}^{\mathrm{c}}$ & $\mathrm{HSE}^{\mathrm{C}}$ \\
\hline \multirow[t]{3}{*}{$\mathrm{V}_{\mathrm{O}}$} & $+/ 0$ & 0.1 & 0.5 & 1.7 & 1.8 & $\ldots$ \\
\hline & $2+/+$ & 1.0 & 1.4 & 2.5 & 2.7 & $\ldots$ \\
\hline & $2+/ 0$ & $\ldots$ & $\ldots$ & 2.1 & 2.2 & 2.2 \\
\hline \multirow[t]{3}{*}{$\mathrm{V}_{\mathrm{Zn}}$} & $-/ 0$ & 0.1 & 0.1 & 0.6 & $\ldots$ & 0.9 \\
\hline & $2-1-$ & 0.3 & 0.4 & 0.6 & $\ldots$ & 2.5 \\
\hline & $2-/ 0$ & $\ldots$ & $\ldots$ & 0.6 & $\ldots$ & $\ldots$ \\
\hline \multirow[t]{6}{*}{$\mathrm{V}_{\mathrm{ZnO}}$} & $2+/ 0$ & $\ldots$ & $\ldots$ & 0.8 & $\ldots$ & $\ldots$ \\
\hline & $2+/+$ & $\ldots$ & $\ldots$ & 0.9 & $\ldots$ & $\ldots$ \\
\hline & $+/ 0$ & $\ldots$ & $\ldots$ & 0.6 & $\ldots$ & $\ldots$ \\
\hline & $-/ 0$ & $\ldots$ & $\ldots$ & 2.4 & $\ldots$ & $\ldots$ \\
\hline & $2-1-$ & $\ldots$ & $\ldots$ & 1.9 & $\ldots$ & $\ldots$ \\
\hline & $2-/ 0$ & $\ldots$ & $\ldots$ & 2.2 & $\ldots$ & $\ldots$ \\
\hline
\end{tabular}

${ }^{\mathrm{a}}$ Ref. 58.

${ }^{\mathrm{b}}$ This work.

${ }^{\mathrm{c}}$ Ref. 78 .

${ }^{\mathrm{d}}$ Ref. 75 .

Transition levels for $\mathrm{V}_{\mathrm{O}}, \mathrm{V}_{\mathrm{Zn}}$, and $\mathrm{V}_{\mathrm{ZnO}}$ vacancies from B3LYP hybrid DFT calculations are given in Table III along with selected literature values. No correction for finite supercell size has been made to our formation energies; transition levels are obtained from relaxed $3 \times 3 \times 3$ supercell formation energies given in Tables I and II. Data in Table III show that uncorrected transition levels from LDA and LDA $+U$ calculations lie well below those predicted by hybrid density functionals. There is good agreement between our transition levels and another recent B3LYP calculation, ${ }^{78}$ which used the same gaussian orbital code and a different method, based on Janak's theorem. ${ }^{79}$ There is also good agreement between our transition levels, obtained using a B3LYP hamiltonian and those by Oba et al. ${ }^{75}$ who used the hybrid functional of Heyd, Scuseria, and Ernzerhof ( $\mathrm{HSE}^{73,74}$ ), except for the position of the $\epsilon(2-/-)$ level of $\mathrm{V}_{\mathrm{Zn}}$. We find that the $\epsilon(-/ 0)$ and $\epsilon(2-/-)$ levels both lie $0.6 \mathrm{eV}$ above the Fermi level, whereas Oba et al. find these levels to lie 0.9 and $2.5 \mathrm{eV}$ above the Fermi level. Thus Oba et al. find a Hubbard $U$ value of $1.6 \mathrm{eV}$ for $\mathrm{V}_{\mathrm{Zn}}$, while we find $0.0 \mathrm{eV}$. This disagreement is surprising since other transition levels in our calculations are in good agreement with other values from hybrid DFT calculations.

\section{Estimate of Hubbard model parameters}

The Hubbard $U$ parameter is obtained from the difference in transition levels of the doubly and singly occupied vacancies, so that for an acceptor defect,

$$
U=\epsilon(2-/-)-\epsilon(-/ 0)=E_{X^{2-}}-2 E_{X^{-}}+E_{X^{0}} .
$$

This method for estimating the values of $U$ includes the relaxation of the lattice and electronic degrees of freedom. The relative contributions of the lattice and electronic relaxations to the $U$ value can be obtained using Eq. (5) with total energies obtained when the lattice has been (or has not been) relaxed. The large defect relaxation energies in $\mathrm{ZnO}$ are associated with large inward or outward movements of ions immediately surrounding the vacancy. For example, the $\mathrm{Zn}$ ions in the $\mathrm{V}_{\mathrm{ZnO}}^{2-}$ vacancy relax inwards (Fig. 1) while the $\mathrm{Zn}$ ions in $\mathrm{V}_{\mathrm{O}}^{2+}$ relax outwards. These relaxations are accompanied by
TABLE IV. Hubbard $U$ parameters in electron volts for unrelaxed and relaxed vacancy defects obtained from transition-level differences. Values given for $\mathrm{V}_{\mathrm{ZnO}}$ relate to negative charge states of the defect.

\begin{tabular}{lcr}
\hline \hline & Unrelaxed & Relaxed \\
\hline $\mathrm{V}_{\mathrm{O}}$ & 1.9 & -0.8 \\
$\mathrm{~V}_{\mathrm{Zn}}$ & 1.5 & 0.0 \\
$\mathrm{~V}_{\mathrm{ZnO}}$ & 1.2 & -0.6 \\
\hline \hline
\end{tabular}

large shifts in single-particle energy eigenvalues in hybrid DFT calculations and large changes in Hubbard $U$ values. Parameter $U$ was also estimated for $\mathrm{V}_{\mathrm{ZnO}}$ using total energy calculations on supercells containing pairs of vacancies. Calculations are performed for different electronic configurations and total energies were fitted to the Hubbard Hamiltonian in Eq. (1).

Hubbard $U$ values from transition-level differences for relaxed and unrelaxed (i.e., bulk equilibrium ion positions) $3 \times 3 \times 3$ supercells are given in Table IV. Unrelaxed defect calculations predict large positive $U$ values for each vacancy defect. The $U$ values for $\mathrm{V}_{\mathrm{Zn}}$ and $\mathrm{V}_{\mathrm{ZnO}}$ unrelaxed vacancies increase by several tenths of an electron volt when extrapolated to infinite defect separation; relaxation of supercells larger than $3 \times 3 \times 3$ using hybrid DFT Hamiltonians is very expensive in computer time and so extrapolation of the relaxed supercell $U$ values to infinite defect separation was not done.

When all atomic positions in the unit cell are allowed to relax, $U$ values for $\mathrm{V}_{\mathrm{O}}$ and $\mathrm{V}_{\mathrm{ZnO}}$ change sign to become negative, while the $U$ value for $\mathrm{V}_{\mathrm{Zn}}$ becomes zero. Relaxation energies given in Table $\mathrm{V}$ were obtained beginning with the energy of the neutral vacancy with atoms in their bulk positions. The neutral vacancy relaxation energy is the amount of energy recovered when all atomic positions in the cell are allowed to relax. The singly charged vacancy relaxation energy is the amount of energy recovered when a charge is added to the neutral supercell, with the relaxed atomic configuration for the neutral defect, and all atomic positions are again allowed to relax. The doubly charged vacancy relaxation energy is obtained by adding a second charge and relaxing again. $\mathrm{V}_{\mathrm{O}}^{2+}$ and $\mathrm{V}_{\mathrm{ZnO}}$ have relaxation energies in excess of $1 \mathrm{eV}$.

The relevance of the relaxation energies in Table $V$ to ferromagnetic exchange in oxides is as follows: if the scattering time is shorter than the lattice relaxation time around a defect which is in a singly negatively charged state, then the vacancy will not relax to its equilibrium doubly charged configuration within the scattering time. The relevant $U$ value in this case is higher than the fully relaxed value by the relaxation energy obtained when a singly charged defect traps a second electron. For example, this would raise the relevant $U$ value for $\mathrm{V}_{\mathrm{ZnO}}$

TABLE V. Relaxation energies in electron volts for vacancy defects.

\begin{tabular}{lccc}
\hline \hline Charge & $\mathrm{V}_{\mathrm{O}}$ & $\mathrm{V}_{\text {Zn }}$ & $\mathrm{V}_{\text {ZnO }}$ \\
\hline 0 & 0.3 & 0.9 & 2.1 \\
1 & 0.8 & 0.4 & 0.6 \\
2 & 1.1 & 0.5 & 0.8 \\
\hline \hline
\end{tabular}


from -0.6 to $+0.2 \mathrm{eV}$ and that for $\mathrm{V}_{\mathrm{Zn}}$ from 0.0 to $+0.5 \mathrm{eV}$, according to the relaxation energies given in Table $\mathrm{V}$.

The predicted negative $U$ character of fully relaxed $\mathrm{V}_{\mathrm{ZnO}}$ in Table IV may arise because its electronic structure is similar to that of $\mathrm{V}_{\mathrm{O}}$, which is also a negative $U$ defect. $\mathrm{V}_{\mathrm{O}}$ has two dangling electron states and $\mathrm{V}_{\mathrm{Zn}}$ has two associated hole states. In $\mathrm{V}_{\mathrm{ZnO}}$, the hole states associated with the $\mathrm{Zn}$ part of the pair vacancy are filled by the dangling electrons from the $\mathrm{O}$ part of the vacancy. $\mathrm{V}_{\mathrm{ZnO}}$ is therefore analogous to $\mathrm{V}_{\mathrm{O}}^{2+}$ and $\mathrm{V}_{\mathrm{ZnO}}^{2-}$ is analogous to $\mathrm{V}_{\mathrm{O}}$. Hence, the electronic structure of $\mathrm{V}_{\mathrm{ZnO}}$ resembles that of $\mathrm{V}_{\mathrm{O}}$ and the positions of the corresponding transition levels are quite similar (Table IV).

Parameter $U$ was also estimated for the unrelaxed $\mathrm{V}_{\mathrm{ZnO}}$ pair vacancy by calculating total energies for different totalspin states when there were three trapped electrons per pair of $\mathrm{V}_{\mathrm{ZnO}}$ vacancies in a $4 \sqrt{ } 3 \times 2 \sqrt{3} \times 2$ supercell; weak antiferromagnetic coupling between electrons was found when there were two electrons in the same supercell, as expected.

Hybrid DFT calculations, with two trapped electrons and the spins in either vacancy in parallel or antiparallel configurations, showed an energy difference for these two states of just $1 \mathrm{meV}$, with the antiparallel configuration lower in energy. Thus there is very weak antiferromagnetic coupling between electrons with one electron per defect with a defect separation of over $11 \AA$. This calculation illustrates the problem of explaining how ferromagnetic exchange between paramagnetic defects can arise over this range. However, similar calculations with three trapped electrons, with a total spin of zero or one-half, resulted in a total energy difference of $220 \mathrm{meV}$, even though the vacancy centers were separated by over $11 \AA$. The spin one-half state was lower in energy. This energy difference is attributed to the difference in Coulomb repulsion energies for the trapped electrons in either state. In the spin-zero state the spin-up and down populations on either site were both 0.75 , while in the spin one-half state the spin-up and down populations were 1.00 and 0.50 for either site. According to the Hubbard model in Eq. (1), the Coulomb energies of the two states are, respectively, $9 U / 8$ and $U$. The total energy difference of $220 \mathrm{meV}$ therefore corresponds to a $U$ value of $1.7 \mathrm{eV}$ for the on-site interaction of trapped electrons, which compares with the value of $U=1.2 \mathrm{eV}$ from the differences in transition levels (Table III).

The value of $t$ for hopping between vacancies is estimated from the bandwidth of the trapped electrons. The bandwidth is $0.4 \mathrm{eV}$ and each vacancy has six neighbors, leading to a $t$ value of $0.07 \mathrm{eV}$; together with a $U$ value of $1.2 \mathrm{eV}$ this gives a $U / W$ ratio of 3 . However, the large value of $U$ in this ratio is found only for the unrelaxed $\mathrm{V}_{\mathrm{ZnO}}$ vacancy.

\section{E. Role of transition-metal impurities}

In order to investigate the magnetic coupling of $\mathrm{Co}^{2+}$ ions in the vicinity of defects with a magnetic moment, a series of calculations was performed in which $\mathrm{Zn}^{2+}$ ions at all unique sites close to $\mathrm{V}_{\mathrm{ZnO}}^{-}$or $\mathrm{V}_{\mathrm{Zn}}^{-}$vacancy centers were individually substituted by $\mathrm{Co}^{2+}$. For each site, calculations were performed with the $\mathrm{Co}^{2+}$ and vacancy-defect electron spins parallel or antiparallel. Calculations were also performed with pairs of $\mathrm{Co}^{2+}$ ions in the absence of vacancy defects to determine near-neighbor direct couplings of $\mathrm{Co}^{2+}$ ions (which
TABLE VI. Total energy difference $\Delta E=E_{\uparrow \uparrow}-E_{\uparrow \downarrow}$ in meV for $\mathrm{Co}_{\mathrm{Zn}}$ separated by distance $\mathrm{d}$ in $\AA$.

\begin{tabular}{lcc}
\hline \hline$d_{\mathrm{Co}-\mathrm{Co}}$ & $\Delta E_{\mathrm{CoCo}}{ }^{\mathrm{a}}$ & $\Delta E_{\mathrm{CoCo}}{ }^{\mathrm{b}}$ \\
\hline 3.20 & 12.2 & 16 \\
4.57 & 0.1 & 6 \\
5.61 & 0.6 & $\cdots$ \\
5.63 & 0.3 & $\cdots$ \\
7.66 & $0.0(2)$ & $\cdots$ \\
\hline
\end{tabular}

aThis work

${ }^{\mathrm{b}}$ Ref. 81.

are all antiferromagnetic, as expected, ${ }^{30,80}$ see Table VI). Chanier et al. ${ }^{81}$ obtain values of 16 and $6 \mathrm{meV}$ for the total energy difference $\Delta E$ using the LSDA $+U$ method for near-neighbor $\mathrm{Co}^{2+}$ ions in the same basal plane and in adjacent planes. These may be compared to our values of 12 and $0.1 \mathrm{meV}$ in Table VI.

When calculations were performed with pairs of $\mathrm{Co}^{2+}$ ions in the presence of the negatively charged defect, it was found that all total energies could be mapped onto an Ising Hamiltonian,

$$
H=\sum_{i<j} J^{\mathrm{Co}_{i} V} S_{\mathrm{Co}_{i}} S_{V}+J^{\mathrm{Co}_{i} \mathrm{Co}_{j}} S_{\mathrm{Co}_{i}} S_{\mathrm{Co}_{j}},
$$

where $J^{\mathrm{Co}_{i} V}$ and $J^{\mathrm{Co}_{i} \mathrm{Co}_{j}}$ are $\mathrm{Co}^{2+} /$ trapped electron and $\mathrm{Co}^{2+} / \mathrm{Co}^{2+}$ coupling energies, respectively. Hamiltonian parameters for $\mathrm{Co}^{2+}$ ions interacting with $\mathrm{V}_{\mathrm{ZnO}}^{-}$or $\mathrm{V}_{\mathrm{Zn}}^{-}$vacancies are given in Table VII as the total energy differences. Most calculations of the difference between ferro- and antiferromagnetic arrangements of the defect and Co ion moments were performed using Co ions substituted at $\mathrm{Zn}$ sites with no lattice relaxation; total energy differences where lattice relaxation was performed are shown in parentheses. Indices $i$ and $j$ label sites around the vacancy and the $\mathrm{Co}^{2+}$ and trapped electron spins $\left(S_{\mathrm{Co}_{i}}\right.$ and $\left.S_{\mathrm{V}}\right)$ are classical unit vectors, so that energy differences between spin parallel and antiparallel configurations are twice the $J$ value. Magnetic coupling of $\mathrm{Co}^{2+}$ ions in $\mathrm{ZnO}$ is antiferromagnetic and very short ranged; it is also antiferromagnetic between single trapped electrons in $\mathrm{V}_{\mathrm{ZnO}}^{-}$vacancies and surrounding $\mathrm{Co}^{2+}$ ions, while it is ferromagnetic between single trapped electrons in $\mathrm{V}_{\mathrm{Zn}}^{-}$and surrounding $\mathrm{Co}^{2+}$ ions.

Strong magnetic coupling between trapped electrons and $\mathrm{Co}^{2+}$ ions is only found where there is significant overlap between the trapped-electron wave function and $\mathrm{Co}^{2+} d$ states. Hence, a trapped electron can form a small magnetic polaron with $\mathrm{Co}^{2+}$ ions in its vicinity, although the range over which strong coupling is found $(>10 \mathrm{meV}$, say) is short and is confined to neighbors within $5 \AA$ of the defect center (Table VII). Even though there is antiferromagnetic coupling of $\mathrm{V}_{\mathrm{ZnO}}^{-}$and $\mathrm{Co}^{2+}$ magnetic moments, the polaron will always have a net moment since the moments of the $\mathrm{Co}^{2+}$ ions ( $\left.3 \mu_{B}\right)$ exceed those of the trapped electron $\left(1 \mu_{B}\right)$ and all $\mathrm{Co}^{2+}$ ions in a polaron have their spins antiparallel to the trapped electron spin. The coupling of $\mathrm{Co}^{2+}$ ions to the trapped electron is stronger than that between $\mathrm{Co}^{2+}$ ions $(6 \mathrm{meV}$ at nearest neighbor and negligible at longer range, Table VI). Most calculations of magnetic coupling between $\mathrm{Co}^{2+}$ ions 
TABLE VII. Total energy difference $\Delta E=E_{\uparrow \uparrow}-E_{\uparrow \downarrow}$ in meV for $\mathrm{V}_{\mathrm{ZnO}}^{-}$and $\mathrm{Co}_{\mathrm{Zn}}$ or $\mathrm{V}_{\mathrm{Zn}}^{-}$and $\mathrm{Co}_{\mathrm{Zn}}$ separated by distance $d$ from the $\mathrm{O}$ or $\mathrm{Zn}$ vacancy site, in angstroms. Values of total energy differences were obtained using bulk atomic positions for all atoms in most cases. Values of total energy differences where all atomic positions in the unit cell were relaxed are given for four cases.

\begin{tabular}{|c|c|c|}
\hline$d_{\mathrm{Co}-\mathrm{V}_{\mathrm{ZnO}}^{-}}$ & $\Delta E_{\mathrm{CoV}_{\mathrm{ZnO}}^{-}}{ }^{\mathrm{a}}$ & $\Delta E_{\mathrm{CoV}_{\mathrm{ZnO}}^{-}}{ }^{\mathrm{b}}$ \\
\hline 1.98 & 394.0 & $\ldots$ \\
\hline 3.25 & 4.6 & 18.9 \\
\hline 3.80 & 1.9 & $\cdots$ \\
\hline 3.80 & -6.4 & $\ldots$ \\
\hline 4.59 & 14.8 & $\ldots$ \\
\hline 4.95 & 0.0 & $\cdots$ \\
\hline 5.00 & 25.4 & 40.4 \\
\hline 5.90 & 1.0 & $\ldots$ \\
\hline 5.95 & 0.3 & $\cdots$ \\
\hline 6.50 & 1.5 & $\ldots$ \\
\hline 6.74 & 2.7 & 2.4 \\
\hline$d_{\mathrm{Co}-V_{\mathrm{Zn}}^{-}}^{-}$ & $\Delta E_{\mathrm{CoV}_{\mathrm{Zn}}^{-}}^{\mathrm{a}}$ & $\Delta E_{\mathrm{CoV}_{\mathrm{Zn}}^{-}}^{\mathrm{b}}$ \\
\hline 3.26 & -78.6 & $\ldots$ \\
\hline 3.30 & 0.4 & $\ldots$ \\
\hline 4.54 & -4.2 & $\ldots$ \\
\hline 5.16 & -8.0 & $\cdots$ \\
\hline 5.59 & 2.2 & $\cdots$ \\
\hline 5.64 & -1.2 & $\ldots$ \\
\hline 5.69 & -8.0 & $\ldots$ \\
\hline 7.63 & 1.4 & 0.4 \\
\hline
\end{tabular}

${ }^{\mathrm{a} B u l k}$ atomic positions.

${ }^{\mathrm{b}}$ Relaxed atomic positions.

and trapped electrons were performed with ions at bulk $\mathrm{ZnO}$ positions because of the computational expense of relaxing atomic positions. However, in four cases (Table VII), all atomic positions in the unit cell were relaxed for both ferroand antiferromagnetic configurations. The magnetic coupling energies determined for these relaxed configurations show some dependence of magnetic coupling energies on atomic position, but do not change the conclusion that small, strongly bound magnetic polarons form around a trapped electron with a radius of about $5 \AA$.

\section{DISCUSSION}

\section{A. Trapped-electron model for ferromagnetism}

The trapped-electron model for ferromagnetism in oxides requires paramagnetic centers which are sufficiently close to permit exchange coupling via electron or hole exchange. It also requires strong interactions between electrons (or holes) and the electron (or hole) at the paramagnetic center. Here, we have adopted the criterion, that the Hubbard model $U$ value to bandwidth $W$ ratio exceeds unity, as indicating likely existence of ferromagnetism in a trapped-electron model.

Paramagnetic vacancy defects, such as F centers or electrons trapped at cation or anion-cation pair vacancies, are observed in many oxides and other ionic compounds; this model can therefore meet a requirement that it be able to explain weak ferromagnetism in a range of materials. The charge state of these centers, and therefore their efficacy in promoting ferromagnetism, will depend on the density of $n$ or $p$-type carriers present in the material and on the transition levels of the particular defect.

The bandwidth for the vacancies considered here is $0.4 \mathrm{eV}$ at a concentration of around 1 at. $\%$. We find values of $t(70 \mathrm{meV})$, $W(400 \mathrm{meV})$ for $\mathrm{V}_{\mathrm{ZnO}}$ vacancies separated by just over $11 \AA$. As noted above, the Curie temperature in the Hubbard model is set by the absolute value of the hopping parameter $t$, and Curie temperatures predicted by HTSE calculations ${ }^{20}$ corresponding to this defect bandwidth lie in the range of 650 to $1460 \mathrm{~K}$. Hence, the requirement that the model supports ferromagnetism above room temperature will be satisfied, provided that the Hubbard $U$ parameter is large enough and that the carrier density providing exchange coupling between electrons trapped at vacancies is in the correct range $\mathrm{e}^{20-22}$ to support ferromagnetism.

We have calculated Hubbard-model $U$ values for the $\mathrm{V}_{\mathrm{ZnO}}$ anion-cation pair vacancy and the $\mathrm{V}_{\mathrm{Zn}}$ cation vacancy. These vacancies have relatively low formation energies in $\mathrm{ZnO}$ for high Fermi-level values, which are likely to be encountered in $n$-type $\mathrm{ZnO}$ (see Fig. 3). $\mathrm{V}_{\mathrm{ZnO}}$ is strongly bound with respect to isolated $V_{Z n}$ and $V_{O}$ single-atom vacancies; at least $\mathrm{V}_{\mathrm{Zn}}$ is expected to be mobile at temperatures used to prepare ferromagnetic $\mathrm{ZnO}$ thin films ${ }^{58}$ and so it is likely that $\mathrm{V}_{\mathrm{ZnO}}$ will exist in these films. The correlation between low oxygen partial pressure and ferromagnetism in $\mathrm{ZnO}$ films would be explained if $\mathrm{V}_{\mathrm{ZnO}}^{-}$(rather than $\mathrm{V}_{\mathrm{Zn}}^{-}$) were the paramagnetic center responsible for ferromagnetism when these centers are coupled to a ferromagnetic state by additional electron carriers.

Using total energy calculations on pairs of $\mathrm{V}_{\mathrm{ZnO}}^{-}$centers in a supercell, we have shown that strong ferromagnetic coupling (of order $200 \mathrm{meV}$ ) between $\mathrm{V}_{\mathrm{ZnO}}^{-}$centers is induced when an extra electron is added to the supercell, provided that the $\mathrm{V}_{\mathrm{ZnO}}$ vacancy structure is unrelaxed, so that the center has a large $U$ value. When the extra coupling electron is absent, only weak antiferromagnetic coupling (of order $1 \mathrm{meV}$ ) is found between the paramagnetic centers. The authors of the present work are not aware of any other first-principles calculations on defect-induced ferromagnetism which consider a noninteger number of electrons per defect. This feature of the model presented here may be key to explaining strong coupling of paramagnetic centers in oxides over ranges exceeding $10 \AA$.

The main difficulty in asserting that $\mathrm{V}_{\mathrm{ZnO}}^{-}$or $\mathrm{V}_{\mathrm{Zn}}^{-}$are the magnetic centers responsible for ferromagnetism in $\mathrm{ZnO}$ is their negative $\left(\mathrm{V}_{\mathrm{ZnO}}\right)$ or small $\left(\mathrm{V}_{\mathrm{Zn}}\right)$ predicted $U$ values, when fully relaxed. However, if we assume that the structures of $\mathrm{V}_{\mathrm{ZnO}}$ and $\mathrm{V}_{\mathrm{Zn}}$ vacancies in the ferromagnetic state are those of $\mathrm{V}_{\mathrm{ZnO}}^{-}$and $\mathrm{V}_{\mathrm{Zn}}^{-}$(because the extra electrons scatter from them in a short time) then their effective $U$ values will be greater than the values obtained using full lattice relaxation in each charge state, by the relaxation energy of the double-charged vacancies. As noted above, the effective $U$ value of $\mathrm{V}_{\mathrm{ZnO}}$ would increase from -0.6 to $+0.2 \mathrm{eV}$ and the value for $\mathrm{V}_{\mathrm{Zn}}$ would change from 0.0 to $+0.5 \mathrm{eV}$. However, these values are too small $\left(\mathrm{V}_{\mathrm{ZnO}}\right.$ ) (or marginal for $\mathrm{V}_{\mathrm{Zn}}$ ) to meet the Hubbard-model criterion for ferromagnetism, $U / W>1$, since $W=0.4 \mathrm{eV}$ at a defect concentration of 1 at.\%. Hubbard $U$ values for $\mathrm{V}_{\mathrm{Zn}}$ from the literature are given in Table III. LDA and LDA $+U^{58}$ 
values for $U$ are 0.2 and $0.3 \mathrm{eV}$, respectively, while a value of $1.6 \mathrm{eV}$ was obtained from a hybrid DFT calculation. ${ }^{75}$ This latter value is in disagreement with our hybrid DFT value of $0.0 \mathrm{eV}$; it arises because the $\epsilon(2-/-)$ level in the calculation by Oba et al. $(2.5 \mathrm{eV})$ is much higher than the value that we obtain $(0.6 \mathrm{eV})$.

\section{B. Comparison to experiment}

The principal source of magnetization in the model presented in Sec. V is charged vacancy defects. This source may be supplemented by transition-metal ion moments which, if present, may be ferro- or antiferromagnetically coupled to carriers associated with the vacancies. We assume a magnetic moment per vacancy of $0.5 \mu_{B}$, the expected moment for $\mathrm{V}_{\mathrm{ZnO}}$ or $\mathrm{V}_{\mathrm{Zn}}$ with 1.5 n-type carriers per vacancy, when fully polarized in an external field. A magnetization of $1 \mathrm{emu} \mathrm{cm}^{-3}$ in $\mathrm{ZnO}$ corresponds to $2.61 \times 10^{-3} \mu_{B} /$ f.u. and the volume per formula unit (f.u.) in $\mathrm{ZnO}$ is $24.2 \AA^{3}$. The mean separation of magnetic vacancy defects with a moment of $0.5 \mu_{B}$ when the bulk magnetization is 1,10 , or $100 \mathrm{emu} \mathrm{cm}^{-3}$ in $\mathrm{ZnO}$ is $16.7,7.7$, or $3.6 \AA$, respectively. We define the mean separation of defects to be the cube root of the volume per defect. The mean defect separation of $\mathrm{V}_{\mathrm{ZnO}}$ pair vacancies in the supercell calculations described in Sec. VD, which were used to obtain the Hubbard $U$ parameter, was $13.2 \AA$ and the shortest defect-defect separation in the supercell was $11.2 \AA$. When this supercell is fully polarized, the magnetization is $1.0 \times 10^{-2} \mu_{B} /$ f.u. or $3.8 \times 10^{-3} \mathrm{emu} \mathrm{cm} \mathrm{cm}^{-3}$. The carrier density corresponding to 1.5 electrons per vacancy in the supercell is $6.5 \times 10^{20} \mathrm{~cm}^{-3}$.

Experimental magnetization data for pure oxide films and films co-doped with transition metals are summarized in Table VIII. Some groups report a nonlinear relationship been magnetization and film thickness, suggesting that the magnetization is concentrated in a surface layer. Indeed, Straumal and coworkers ${ }^{36}$ have surveyed the experimental literature

TABLE VIII. Magnetic moments measured in undoped and TMdoped oxide single crystals, films, and wires. Film thickness or wire diameter, preparation method, magnetization converted to $\mu_{B} /$ f.u., and the temperature at which magnetization was measured are given.

\begin{tabular}{lcclcc}
\hline \hline System & Reference & Thick. (nm) & Method & $\mu_{B} /$ f.u. & $T(\mathrm{~K})$ \\
\hline $\mathrm{ZnO}$ & 10 & 375 & PLD & $5.2 \times 10^{-3}$ & 300 \\
$\mathrm{ZnO}$ & 10 & 10,50 & PLD & $5.2 \times 10^{-1}$ & 300 \\
$\mathrm{ZnO}$ & 83 & 50 & Zn nanowire & $2.6 \times 10^{-3}$ & 300 \\
$\mathrm{ZnO}$ & 84 & 10 & hydrothermal & $5.7 \times 10^{-5}$ & 300 \\
$\mathrm{ZnO}$ & 85 & unknown & PLD & $2.1 \times 10^{-3}$ & 290 \\
$\mathrm{ZnO}$ & 36 & $100-550$ & hydrothermal & $0.8 \times 10^{-3}$ & 300 \\
$\mathrm{ZnO}$ & 19 & unknown & crystal & $0.3 \times 10^{-3}$ & 300 \\
$\mathrm{ZnO} / \mathrm{Mn}$ & 36 & unknown & hydrothermal & $2.0 \times 10^{-3}$ & 300 \\
$\mathrm{ZnO} / \mathrm{Co}$ & 3 & $60-120$ & PLD & 0.26 & 300 \\
$\mathrm{ZnO} / \mathrm{Co}$ & 7 & 50 & PLD & 0.14 & 300 \\
$\mathrm{ZnO} / \mathrm{Co}$ & 5 & $100-500$ & PLD & 0.03 & 300 \\
$\mathrm{TiO}_{2}$ & 12 & 200 & PLD & $5.2 \times 10^{-2}$ & 300 \\
$\mathrm{TiO}_{2}$ & 13 & 200 & PLD & 0.14 & 300 \\
$\mathrm{HfO}_{2}$ & 12 & 200 & PLD & 0.11 & 300 \\
$\mathrm{HfO}_{2}$ & 82 & unknown & PLD & 0.15 & 300 \\
\hline \hline
\end{tabular}

and shown that there are critical grain-boundary-to-volume ratios, above which ferromagnetism is observed in $\mathrm{ZnO}$ or Mn-doped $\mathrm{ZnO}$. Hong and coworkers ${ }^{10}$ report a saturation magnetization of $2 \mathrm{emu} \mathrm{cm}^{-3}\left(5.2 \times 10^{-3} \mu_{B} /\right.$ f.u. $)$ in $375-\mathrm{nm}$ $\mathrm{ZnO}$ films at $300 \mathrm{~K}$ and over $200 \mathrm{emu} \mathrm{cm}^{-3}\left(0.52 \mu_{B} /\right.$ f.u. $)$ at $300 \mathrm{~K}$ in 10 - or $50-\mathrm{nm}$ films produced by pulsed laser deposition (PLD). The same group ${ }^{12}$ reported a saturation magnetization of $15 \mathrm{emu} \mathrm{cm}^{-3}\left(5.2 \times 10^{-2} \mu_{B} /\right.$ f.u. $)$ in 200 $\mathrm{nm} \mathrm{TiO}_{2}$ films and $30 \mathrm{emu} \mathrm{cm} \mathrm{cm}^{-3}\left(0.11 \mu_{B} /\right.$ f.u. $)$ in $200-\mathrm{nm}$ $\mathrm{HfO}_{2}$ films. Venkatesan and coworkers ${ }^{82}$ reported a saturation magnetization of $0.15 \mu_{B} /$ f.u. in $\mathrm{HfO}_{2}$ in films between 45 and $135 \mathrm{~nm}$ thick. Sudakar and coworkers ${ }^{13}$ reported a saturation magnetization of $40 \mathrm{emu} \mathrm{cm}^{-3}\left(0.12 \mu_{B} /\right.$ f.u. $)$ in sputter deposited and vacuum annealed $\mathrm{TiO}_{2}$ films and a magnetization of around $5 \mathrm{emu} \mathrm{cm} \mathrm{cm}^{-3}\left(1.9 \times 10^{-2} \mu_{B} /\right.$ f.u. $)$ in $100-n m$ films. Recently, Khalid and coworkers ${ }^{19}$ have used a range of techniques on a range of oxides to measure the level of ferromagnetic impurities. They find a ferromagnetic contribution that persists to room temperature and that cannot be accounted for by the levels of (mainly transition metal) ferromagnetic impurities found. However, if these impurities are not taken into account, the ferromagnetic moment, which is not due to impurities, can be greatly overestimated.

The threshold carrier density at which ferromagnetism is first observed by Behan and coworkers ${ }^{5}$ in the metallic regime in Co- and Al-doped $\mathrm{ZnO}$ films is $8 \times 10^{19} \mathrm{~cm}^{-3}$; the moment is greatest at a carrier density of $7 \times 10^{20} \mathrm{~cm}^{-3}$ in films with up to $2 \% \mathrm{Al}$ co-doping. If vacancy filling for maximum ferromagnetic moment is 1.5 electrons per vacancy (assuming that the Fermi level lies below the conduction band minimum) the mean defect separation at the threshold for ferromagnetism is $26.6 \AA$ and is $12.9 \AA$ at the carrier density at which the maximum ferromagnetic moment is observed.

The defect concentrations assumed in the model presented here can reasonably account for ferromagnetism in oxide films with a bulk magnetization in the range 1 to $10 \mathrm{emu} \mathrm{cm}^{-3}$ $\left(2.6 \times 10^{-3}\right.$ to $2.6 \times 10^{-2} \mu_{B} /$ f.u., mean defect separation 16.7 to $7.7 \AA$ ) and corresponding carrier densities for greatest magnetization in the range $3 \times 10^{20}$ and $3 \times 10^{21} \mathrm{~cm}^{-3}$. This range coincides with the carrier density at which Behan and coworkers observe the greatest magnetization $(6 \times$ $\left.10^{20} \mathrm{~cm}^{-3}\right)$. When transition-metal dopant ions are present and couple to vacancy defect electrons, the range may extend upwards from this range as the moment per defect may be up to five times larger. Thus the vacancy-defect model that we propose is compatible with the observed magnetization and carrier density ranges in which ferromagnetism is reported by some groups (Table VIII). For magnetizations below 1 emu cm ${ }^{-3}\left(2.6 \times 10^{-3} \mu_{B} /\right.$ f.u. $)$ defects will be too far apart to support a high Curie temperature, since the bandwidth $W$ will be small. However, in such cases an inhomogeneously magnetized sample can be postulated. However, it is more difficult to see how an interacting defect model can account for the very high magnetizations $\left(>0.1 \mu_{B} /\right.$ f.u. $)$ observed in some cases $\left(0.5 \mu_{B} /\right.$ f.u. in $\mathrm{ZnO},{ }^{10} 0.14 \mu_{B} /$ f.u. in $\mathrm{TiO}_{2},{ }^{13}$ or $0.14 \mu_{B}$ /f.u. in $\mathrm{HfO}_{2}{ }^{82}$ ). In the former case, each $\mathrm{Zn}$ site in the lattice would have to have a moment equal to the proposed $0.5 \mu_{B}$ per vacancy. For magnetizations significantly above 10 emu $\mathrm{cm}^{-3}\left(2.6 \times 10^{-2} \mu_{B} /\right.$ f.u. $)$ defects will be so close that crystalline order in the films may not be observed. Such 
high moments might, of course, be explained by the presence of ferromagnetic transition-metal impurities. ${ }^{19}$

\section{Comparison to other models}

Theoretical models for ferromagnetism in oxide films have focused on transition-metal-doped oxide films. Almost all models or first-principles calculations which find a ferromagnetic state to be energetically preferable find that $n$-type carriers are necessary and that coupling extends only over a short range (up to second nearest neighbor). One experimental and theoretical study of Li-doped $\mathrm{ZnO}$ found ferromagnetism in $p$-type $\mathrm{ZnO} .^{52}$ The donor impurity band exchange model from Coey and coworkers ${ }^{26}$ uses a Kondo Hamiltonian to predict a Curie temperature for transition-metal impurities in metal oxides, $T_{C}$. The exchange integral $J$ was assumed to be $1.5 \mathrm{eV}$ and to favor (anti-)ferromagnetic coupling when the transition metal $d$ band is (more)less than half-full. The resulting expression for $T_{C}$ [Eq. (6) in Ref. 26] predicts $T_{C} \sim$ $18 \mathrm{~K}$ for a $10 \%$ impurity spin doping level and finds that significant (resonant) enhancement of the probability density of the donor electron (by 25 to 50 times) at the transition metal is needed for $T_{C} \sim 500 \mathrm{~K} .{ }^{26}$ Pemmaraju and coworkers ${ }^{27}$ find strong ferromagnetic coupling between $\mathrm{Co}_{\mathrm{Zn}} / \mathrm{V}_{\mathrm{O}}$ pairs separated by up to $\sim 6$ Åusing a self-interaction-corrected DFT method. They report classical Monte Carlo simulations for a Heisenberg model with a magnetic anisotropy term and predict $T_{C} \sim 250 \mathrm{~K}$. They also find the strongest ferromagnetic coupling between pairs of $\mathrm{Co}_{\mathrm{Zn}} / \mathrm{V}_{\mathrm{O}}$ defect complexes is greatest when there is one electron per pair.

There have been several similar reports of ferromagnetic coupling between transition-metal magnetic moments induced by $n$-type carriers in the $\mathrm{ZnO} / \mathrm{Co}$ system. Lee and Chang ${ }^{47}$ using a GGA-DFT method find a strong ferromagnetic interaction $(\sim 80 \mathrm{meV})$ between nearest-neighbor Co ions when there is one electron per Co ion. Kan and coworkers ${ }^{48}$ used hybrid DFT calculations similar to those used in this work to show that nearest-neighbor $\mathrm{Co}^{2+}$ ions have a strong ferromagnetic interaction $(\sim 90 \mathrm{meV})$ when one electron per Co is introduced into a $2 \times 2 \times 2$ supercell, corresponding to a carrier density $\left(5.0 \times 10^{21} \mathrm{~cm}^{-3}\right)$ much higher than carrier densities at which ferromagnetism is observed in experiment. ${ }^{5}$ They do not find ferromagnetic coupling at lower carrier densities; in this work, we found negligible magnetic coupling $(<1 \mathrm{meV})$ when two Co ions are substituted in a $3 \times 3 \times 2$ supercell, separated by $7 \AA$, and one electron is added to the cell. The carrier density in this case is $1.1 \times 10^{21} \mathrm{~cm}^{-3}$. Walsh and coworkers ${ }^{30}$ used DFT and DFT $+U$ methods and found strong ferromagnetic coupling ( $\sim 350 \mathrm{meV})$ between nearest-neighbor Co ion pairs only when a Hubbard $U$ term was added to the Hamiltonian for both $\mathrm{Zn}-3 d$ and $\mathrm{Zn}-4 s$ atomic states, otherwise they found antiferromagnetic coupling. Adding this term to both atomic states caused minority-spin Co- $3 d$ levels, which were empty in the absence of extra carriers, to populate before states at the $\mathrm{ZnO}$ conduction band minimum (CBM) when $n$-type carriers are introduced. Lany and coworkers ${ }^{49}$ using DFT-GGA and DFT + nonlocal-external-potential (NLEP) methods found similar results to Walsh and coworkers. ${ }^{30}$ They found that DFT in a GGA approximation predicts a weak antiferromagnetic interaction when there is one $n$-type carrier per Co ion pair and a strong ferromagnetic interaction $(\sim 300 \mathrm{meV})$ for the DFT+NLEP calculation. The reason for the different behavior in the two methods is the predicted occupancy of the Co-3d levels, which are empty in the absence of $n$-type carriers.

It is clear that ferromagnetism with a $T_{C}$ value greater than room temperature is predicted by these calculations if empty Co-3d levels are populated rather than the conduction band, when carriers are added to $\mathrm{ZnO} / \mathrm{Co}$ in a concentration range where there is one to two carriers per Co dopant ion. Empirically, it was found that the magnetization was greatest in $\mathrm{ZnO}$ films with $5 \% \mathrm{Co}$ when there were 0.3 carriers per Co $\left(6 \times 10^{20} \mathrm{~cm}^{-3}\right)$. However, a careful study of $\mathrm{ZnO} / \mathrm{Co}$, in which a connection between ferromagnetism and $n$-type carriers was sought, found only very weak ferromagnetism ( 0.001 to $0.06 \mu_{B}$ per Co ion) in epitaxially grown thin films of high crystallinity. ${ }^{46}$ This corresponds to a magnetization of $8.0 \times 10^{-5}$ to $4.8 \times 10^{-3} \mu_{B}$ /f.u.. A moment of $0.03 \mu_{B}$ per Co ion $\left(2.4 \times 10^{-3} \mu_{B} /\right.$ f.u. $)$ was found in films with an $n$-type carrier density of $5.4 \times 10^{20} \mathrm{~cm}^{-3}$. ${ }^{46}$ This suggests that empty $3 d$ levels do not become populated when carrier densities of this order are present in pristine $\mathrm{ZnO} / \mathrm{Co}$ films.

In the hybrid DFT calculations reported in Table IV, we find a strong $s d$ anti-ferromagnetic coupling $(\sim 200 \mathrm{meV})$ between a trapped electron in $\mathrm{V}_{\mathrm{ZnO}}^{-}$and a neighboring $\mathrm{Co}$ ion. This falls rapidly with distance, as expected for a nonresonant interaction, and is negligible for distances between the defect center and the Co ion greater than $5 \AA$. We find a weaker $p d$ ferromagnetic coupling $(\sim 40 \mathrm{meV})$ between a trapped electron in a $\mathrm{V}_{\mathrm{Zn}}^{-}$vacancy and a Co ion, which also falls off rapidly with distance. The short-range couplings between $\mathrm{V}_{\mathrm{ZnO}}^{-}$and $\mathrm{V}_{\mathrm{Zn}}^{-}$and neighboring Co ions arise because the empty Co $3 d$ levels are predicted by the hybrid DFT method to lie around $2 \mathrm{eV}$ above the CBM. ${ }^{37}$

\section{SUMMARY}

Ferromagnetism at ambient temperature has been reported in a wide range of oxide films and single crystals both transition-metal doped and undoped and cannot be accounted for in terms of magnetic impurities alone. Paramagnetic $F$ centers and negatively charged anion vacancies and anioncation pair vacancies are commonly observed in a range of oxides and other ionic compounds via EPR; the model considered here consists of such centers exchange coupled to a ferromagnetic state via extra carriers, which are present through doping, etc.

This model for ferromagnetism requires vacancy defects which trap one or two electrons and an $n$-type carrier density. The vacancy density is assumed to be of order 1 at.\%. It is assumed that such a system can be represented by a one-band Hubbard model in which the model sites are vacancies and hopping occurs between sites with amplitude $t$ and the onsite Hubbard repulsion energy is $U$. Short reviews of relevant experimental and theoretical literature are given.

Using hybrid DFT calculations on large supercells containing two unrelaxed vacancies, we find weak antiferromagnetic coupling between electrons trapped in $\mathrm{V}_{\mathrm{ZnO}}$ defects separated by over $10 \AA$, as expected. However, when the number of electrons is increased to 1.5 per $\mathrm{V}_{\mathrm{ZnO}}$ vacancy, we find a strong ferromagnetic coupling of over $200 \mathrm{meV}$. This strong 
coupling is due to exchange of the extra electron without adiabatic relaxation of the vacancy as the exchanged electron is scattered.

Hybrid DFT calculations were used to estimate Hubbard model parameters for $\mathrm{V}_{\mathrm{O}}, \mathrm{V}_{\mathrm{Zn}}$, and $\mathrm{V}_{\mathrm{ZnO}}$ vacancies with relaxed and unrelaxed atomic positions. Large positive $U$ values are found for these vacancies when unrelaxed atomic positions are used; when atomic positions, which were fully relaxed for each charge state are used instead, the $U$ values for $\mathrm{V}_{\mathrm{O}}$ and $\mathrm{V}_{\mathrm{ZnO}}$ become negative, while that for $\mathrm{V}_{\mathrm{Zn}}$ becomes zero. The effective $U$ value for scattering of an electron by a paramagnetic, singly negatively charged $\mathrm{V}_{\mathrm{Zn}}^{-}$or $\mathrm{V}_{\mathrm{ZnO}}^{-}$may be that obtained using atomic positions which were relaxed for the singly negatively charged state. In that case the $U$ values for $\mathrm{V}_{\mathrm{ZnO}}$ and $\mathrm{V}_{\mathrm{Zn}}$ are +0.2 and $+0.5 \mathrm{eV}$, respectively.

While strong ferromagnetic coupling over considerable distance is found for certain electron fillings from total energy calculations, relaxation of paramagnetic defects in $\mathrm{ZnO}\left(\mathrm{V}_{\mathrm{ZnO}}^{-}\right.$ and $\mathrm{V}_{\mathrm{Zn}}^{-}$) results in large changes in $U$ values, from large and positive to small (positive or negative). The simple criterion for ferromagnetism in a Hubbard model, that the ratio $U / W$ exceeds unity, is not met for these defects in their equilibrium state and the model does not predict ferromagnetism in $\mathrm{ZnO}$.

Magnetic coupling between electrons trapped in $\mathrm{V}_{\mathrm{ZnO}}$ or $\mathrm{V}_{\mathrm{Zn}}$ vacancies and magnetic moments of nearby Co ions in $\mathrm{Zn}$ substitutional sites is investigated. Strong, short-ranged antiferromagnetic coupling is found between $\mathrm{V}_{\mathrm{ZnO}}^{-}$and $\mathrm{Co}^{2+}$ and strong, short-ranged ferromagnetic coupling is found between $\mathrm{V}_{\mathrm{Zn}}^{-}$and $\mathrm{Co}^{2+}$. Strong coupling is found when the vacancy and $\mathrm{Co}^{2+}$ ion are close neighbors and the defect-electron wave function overlaps the $\mathrm{Co}^{2+}$ ion. The interaction is nonresonant and therefore decays rapidly in space.

\section{ACKNOWLEDGMENTS}

A.C. is grateful to Trinity College for a postgraduate studentship. Computer time was provided by the Irish Centre for High End Computing and the Trinity Centre for High Performance Computing, which are supported under the Irish National Development Plan. The project was supported by Science Foundation Ireland under Grant No. 09/RFP/MTR2295. The authors acknowledge helpful discussions with Gillian Gehring, Robert Barklie, Cormac McGuinness, and Michael Coey.

\section{APPENDIX A: DETAILS OF CALCULATIONS}

All calculations were performed using the CRYSTAL program. ${ }^{67}$ The Gaussian orbital basis for $\mathrm{Zn}^{86}$ is the basis previously used by Jaffe and coworkers. ${ }^{86}$ Different $\mathrm{O}$ basis sets were used for $\mathrm{O}$ in LDA or B3LYP calculations. The basis previously used by Valenzano and coworkers ${ }^{87}$ was used in LDA calculations and the basis previously used by Towler and coworkers ${ }^{88}$ was used for B3LYP calculations. Calculations in this work were performed using the Dirac exchange approximation $^{69}$ and the Vosko-Wilks-Nusair approximation to correlation $^{70}$ in the LDA Hamiltonian. The Becke-3 Lee, Yang, and Parr (B3LYP) hybrid density functional ${ }^{89,90}$ contains
Hartree-Fock exchange with weight $A$, the local density approximation to exchange $\mathrm{E}_{x}^{\mathrm{LDA}}$ with weight $(1-A){ }^{69}$ Becke's gradient corrected exchange functional $\mathrm{E}_{x}^{\mathrm{Becke}}$ with weight $B,{ }^{71}$ the Lee, Yang, and Parr approximation to the correlation functional $\mathrm{E}_{c}^{\mathrm{LYP}}$ with weight $C{ }^{72}$ and the Vosko, Wilks, and Nusair approximation to the electron correlation functional $\mathrm{E}_{c}^{\mathrm{VWN}}$ with weight $(1-C):^{70}$

$$
\begin{aligned}
E_{\mathrm{xc}}= & (1-A)\left(E_{x}^{\mathrm{LDA}}+B E_{x}^{\mathrm{Becke}}\right)+A E_{x}^{\mathrm{HF}} \\
& +(1-C) E_{c}^{\mathrm{VWN}}+C E_{c}^{\mathrm{LYP}} .
\end{aligned}
$$

The B3LYP hybrid density functional, with $A(=0.2), B$, and $C$ fixed at their canonical values, is used throughout this work.

A $6 \times 6 \times 6$ Monkhorst-Pack net was used for selfconsistent field calculations; a denser $12 \times 12 \times 12$ net was used for total energy evaluations. It was necessary to use this high $k$-point density to obtain converged total energies for metallic states that are encountered when an electron is added to (or removed from) the perfect bulk cell in transition level calculations. The reason for calculating the energies of these states is explained in Appendix B. Tolerances for lattice sum convergence within the CRYSTAL program were chosen to be 8 , $8,8,8$, and 16. Wave-function amplitude plots in Fig. 2 were generated using the XCRYSDEN program. ${ }^{91,92}$

\section{APPENDIX B: CHARGED-DEFECT CALCULATIONS}

Methods for calculating formation energies of charged defects have been described elsewhere ${ }^{65}$ and applied using both plane-wave codes ${ }^{49,58}$ and the CRYSTAL Gaussian orbital code. ${ }^{78,93}$ Here, we give details of the methods used to calculate defect transition levels and Hubbard $U$ values (see Table IX). In particular, we address the problems of obtaining total energy differences for cells with different net charges, including defect interaction with compensating charge backgrounds, and how transition levels are affected by lattice relaxation.

According to Eq. (2), the difference in total energy when a supercell containing a defect becomes charged is

$$
E_{X^{q}}^{f}-E_{X^{0}}^{f}=E_{X^{q}}-E_{X^{0}}+q\left(E_{F}-E_{V}\right) .
$$

This difference is zero when the Fermi level is at the defect transition level. $\mathrm{A} \epsilon(0 /+)$ transition level can also be calculated

TABLE IX. Transition levels and Hubbard $U$ parameters in electron volts for unrelaxed and relaxed vacancy defects in $L \times L \times$ $L$ supercells.

\begin{tabular}{cccccc}
\hline \hline & & $\begin{array}{c}L=3 \\
\text { relaxed }\end{array}$ & $U$ & $\begin{array}{c}L=3 \\
\text { unrelaxed }\end{array}$ & $U$ \\
\hline $\mathrm{V}_{\mathrm{O}}$ & $+/ 0$ & 1.7 & -0.8 & 2.2 & 1.9 \\
& $2+/+$ & 2.5 & $\ldots$ & 0.3 & $\cdots$ \\
& $2+/ 0$ & 2.1 & $\ldots$ & 1.2 & $\ldots$ \\
$\mathrm{V}_{\mathrm{Zn}}$ & $-/ 0$ & 0.6 & 0.0 & 0.4 & 1.5 \\
& $2-/-$ & 0.6 & $\ldots$ & 1.9 & $\cdots$ \\
& $2-/ 0$ & 0.6 & $\ldots$ & 1.8 & $\cdots$ \\
$\mathrm{V}_{\mathrm{ZnO}}$ & $-/ 0$ & 2.4 & -0.6 & 2.0 & 1.2 \\
& $2-/-$ & 1.9 & $\cdots$ & 3.2 & $\cdots$ \\
& $2-/ 0$ & 2.1 & $\cdots$ & 1.0 & $\cdots$ \\
\hline \hline
\end{tabular}


from the change in energy when a hole is created in a bulk crystal supercell and transferred to a similar supercell with a defect. The energetic costs of these steps are $E_{\mathrm{bulk}^{+}}-E_{\mathrm{bulk}^{0}}$ and $E_{X^{+}}-E_{X^{0}}+E_{\mathrm{bulk}^{0}}-E_{\mathrm{bulk}^{+}}$, where $E_{\mathrm{bulk}^{0}}$ and $E_{\mathrm{bulk}^{+}}$are the total energies of the neutral bulk supercell or the bulk supercell with one electron removed and $E_{X^{0}}$ and $E_{X^{+}}$are total energies for similar supercells with neutral or $1+$ charge states. The latter sum of terms is the Fermi-level position relative to the top of the valence band at which the defect changes charge state and consists of differences in total energies of supercells with equal net charge states,

$$
E_{X^{+}}-E_{\text {bulk }^{+}}-\left(E_{X^{0}}-E_{\text {bulk }^{0}}\right) .
$$

The energy difference $E_{\text {bulk+ }}-E_{\text {bulk }^{0}}$ is equal to $E_{V}$, the absolute position of the valence band edge from the hybrid DFT calculation. Its value for bulk $\mathrm{ZnO}$ in this work is $+7.36 \mathrm{eV}$, which indicates that the position of the valence band maximum is above the vacuum level, as is usually the case for density-functional calculations on solids. This value may be compared to a value of $+7.14 \mathrm{eV}$ found for $\mathrm{CuGaS}_{2}{ }^{93}$ using the same electronic-structure code. Obtaining a converged result for the value of $\mathrm{E}_{V}$ requires a high $k$-point density, even for large supercells. A $12 \times 12 \times 12$ Gilat net was used for all total energy evaluations on $3 \times 3 \times 3$ supercells containing 108 ions. The position of the conduction band edge is obtained from the energy difference, $E_{\text {bulk }}^{-}-E_{\text {bulk }}^{0}=+11.06 \mathrm{eV}$. The band gap obtained this way is $3.70 \mathrm{eV}$. This value lies above the value obtained from the difference in single-particle energy eigenvalues at the $\Gamma$ point $(3.26 \mathrm{eV})$, as expected, ${ }^{49}$ and is in reasonable agreement with the experimental value $3.47 \mathrm{eV}^{25}$ Transition levels for negatively charged defects are referred to the valence band maximum using the band gap obtained from the difference in ionization potential and electron affinity, $3.70 \mathrm{eV}$

Although transition levels determined using Eq. (B2) are derived from energy differences between supercells with the same net charge, transition levels still depend on the lattice constant $L$. This has been discussed by Makov and Payne ${ }^{68}$ and others. ${ }^{49,93,94}$ The energy of a localized charge distribution immersed in a compensating background charge ${ }^{68}$ is

$$
\Delta E=\frac{q^{2} \alpha}{2 \epsilon L}+\frac{2 \pi q Q}{3 \epsilon L^{3}}+O\left(L^{-5}\right),
$$

where $\alpha$ is the Madelung constant, $\epsilon$ is the relative permittivity for the bulk material, $q$ is the net charge, and $Q$ is the quadrupole moment of the charge distribution. It is generally acknowledged that the term containing $1 / L$ in Eq. (B3) overestimates the dependence of the transition-level position on separation of defect centers $L$, so the dependence on supercell size must be calculated for each vacancy defect in each charge state. It is also important to note that in a real $n$-type sample, compensating counter-ions will make a contribution to the Madelung potential of an electron trapped in a vacancy very similar to that of a uniform compensating background charge. Extrapolation of the transition-level position to infinite dilution is appropriate for crystals with a low defect density, but in high defect densities and, therefore, in the model being considered here, transition-level positions for a specific, finite defect density are more appropriate.

In order to estimate the dependence of transition levels on defect separation, total energy calculations were performed on $2 \times 2 \times 2,3 \times 3 \times 3$, and $4 \times 4 \times 4$ supercells with $\mathrm{V}_{\mathrm{O}}$, $\mathrm{V}_{\mathrm{Zn}}$, and $\mathrm{V}_{\mathrm{ZnO}}$ vacancy defects as well as corresponding bulk supercells. Vacancy-defect calculations were performed for each relevant charge state of the vacancy and bulk supercell calculations were performed with an extra electron, one hole and the neutral bulk. Relaxation of atomic positions was not performed in these calculations because of the relatively high computational expense of hybrid DFT calculations. Only $3 \times 3 \times 3$ supercells containing vacancies in each charge state were relaxed in order to obtain relaxation energies. A good fit is obtained when differences in total energies of supercells, $E_{X^{2-}}-E_{X^{-}}$and $E_{X^{-}}-E_{X^{0}}$, containing $\mathrm{V}_{\mathrm{Zn}}$ and $\mathrm{V}_{\mathrm{ZnO}}$ vacancies are fitted to the term in $L^{-1}$ in Eq. (B3) for the three supercell sizes mentioned above. There are insufficient data points to obtain a fit including the $L^{-3}$ term in Eq. (B3).
${ }^{1}$ K. Ueda, H. Tabata, and T. Kawai, Appl. Phys. Lett. 79, 988 (2001). ${ }^{2}$ J. M. D. Coey, A. P. Douvalis, C. B. Fitzgerald, and M. Venkatesan, Appl. Phys. Lett. 84, 1332 (2004).

${ }^{3}$ M. Venkatesan, C. B. Fitzgerald, J. G. Lunney, and J. M. D. Coey, Phys. Rev. Lett. 93, 177206 (2004).

${ }^{4}$ J. R. Neal, A. J. Behan, R. M. Ibrahim, H. J. Blythe, M. Ziese, A. M. Fox, and G. A. Gehring, Phys. Rev. Lett. 96, 197208 (2006).

${ }^{5}$ A. J. Behan, A. Mokhtari, H. J. Blythe, D. Score, X.-H. Xu, J. R. Neal, A. M. Fox, and G. A. Gehring, Phys. Rev. Lett. 100, 047206 (2008).

${ }^{6}$ A. Barla, G. Schmerber, E. Beaurepaire, A. Dinia, H. Bieber, S. Colis, F. Scheurer, J.-P. Kappler, P. Imperia, F. Nolting, F. Wilhelm, A. Rogalev, D. Muller, and J. J. Grob, Phys. Rev. B 76, 125201 (2007).

${ }^{7}$ T. Tietze, M. Gacic, G. Schutz, G. Jakob, S. Bruck, and E. Goering, New J. Phys. 10, 055009 (2008).

${ }^{8}$ T. Dietl, Nat. Mater. 9, 965 (2010).
${ }^{9}$ J. M. D. Coey, M. Venkatesan, P. Stamenov, C. B. Fitzgerald, and L. S. Dorneles, Phys. Rev. B 72, 024450 (2005).

${ }^{10}$ N. H. Hong, J. Sakai, and V. Brizé, J. Phys. Condens. Matter 19, 036219 (2007).

${ }^{11}$ S. Banerjee, M. Mandal, N. Gayathri, and M. Sardar, Appl. Phys. Lett. 91, 182501 (2007).

${ }^{12}$ N. H. Hong, J. Sakai, and F. Gervais, J. Magn. Magn. Mater. 316, 214 (2007).

${ }^{13}$ C. Sudakar, P. Kharel, R. Suryanarayanan, J. S. Thakur, V. M. Naik, R. Naik, and G. Lawes, J. Magn. Magn. Mater. 320, L31 (2008).

${ }^{14}$ H. Hsu, J. C. A. Huang, Y. H. Huang, Y. F. Liao, M. Z. Lin, C. H. Lee, J. F. Lee, S. F. Chen, L. Y. Lai, and C. P. Liu, Appl. Phys. Lett. 88, 242507 (2006).

${ }^{15}$ C. Sudakar, P. Kharel, G. Lawes, R. Suryanarayanan, R. Naik, and V. M. Naik, J. Phys. Condens. Matter 19, 026212 (2007).

${ }^{16}$ X.-C. Liu et al., J. Phys. Condens. Matter 20, 025208 (2008). 
${ }^{17}$ J. M. D. Coey, P. Stamenov, R. D. Gunning, M. Venkatesan, and K. Paul, New. J. Phys. 12, 053025 (2010).

${ }^{18}$ A. Zunger, S. Lany, and H. Raebiger, Physics 3, 53 (2010).

${ }^{19}$ M. Khalid, A. Setzer, M. Ziese, P. Esquinazi, D. Spemann, A. Poppl, and E. Goering, Phys. Rev. B 81, 214414 (2010).

${ }^{20}$ J. A. Henderson, J. Oitmaa, and M. C. B. Ashley, Phys. Rev. B 46, 6328 (1992)

${ }^{21}$ T. Herrmann and W. Nolting, J. Magn. Magn. Mater. 170, 253 (1997).

${ }^{22}$ M. Ulmke, Eur. Phys. J. B 1, 301 (1998).

${ }^{23}$ J. Wahle, N. Blümer, J. Schlipf, K. Held, and D. Vollhardt, Phys. Rev. B 58, 12749 (1998).

${ }^{24}$ W. Nolting and W. Borgiel, Phys. Rev. B 39, 6962 (1989).

${ }^{25}$ Data in Science and Technology; Semiconductors Other than Group IV Elements and III-V Compounds, edited by O. Madelung (Springer-Verlag, Berlin, 1992), pp. 26 and 27.

${ }^{26}$ J. M. D. Coey, M. Venkatesan, and C. B. Fitzgerald, Nat. Mater. 4, 173 (2005).

${ }^{27}$ C. D. Pemmaraju, R. Hanafin, T. Archer, H. B. Braun, and S. Sanvito, Phys. Rev. B 78, 054428 (2008).

${ }^{28}$ A. Kaminski and S. Das Sarma, Phys. Rev. Lett. 88, 247202 (2002).

${ }^{29}$ A. C. Durst, R. N. Bhatt, and P. A. Wolff, Phys. Rev. B 65, 235205 (2002).

${ }^{30}$ A. Walsh, J. L. Da Silva, and S.-H. Wei, Phys. Rev. Lett. 100, 256401 (2008).

${ }^{31}$ I. S. Elfimov, S. Yunoki, and G. A. Sawatzky, Phys. Rev. Lett. 89, 216403 (2002).

${ }^{32}$ D. P. Norton et al., Appl. Phys. Lett. 83, 5488 (2003).

${ }^{33}$ S. C. Wi et al., Appl. Phys. Lett. 84, 4233 (2004).

${ }^{34}$ A. C. Tuan et al., Phys. Rev. B 70, 054424 (2004).

${ }^{35}$ E. Biegger, M. Fonin, U. Rüdiger, N. Janssen, M. Beyer, T. Thomay, R. Bratschitsch, and Y. S. Dedkov, J. Appl. Phys. 101, 073904 (2007).

${ }^{36}$ B. B. Straumal, A. A. Mazilkin, S. G. Protasova, A. A. Myatiev, P. B. Straumal, G. Schütz, P. A. van Aken, E. Goering, and B. Baretzky, Phys. Rev. B 79, 205206 (2009).

${ }^{37}$ C. H. Patterson, Phys. Rev. B 74, 144432 (2006).

${ }^{38}$ M. Gacic, G. Jakob, C. Herbort, H. Adrian, T. Tietze, S. Brück, and E. Goering, Phys. Rev. B 75, 205206 (2007).

${ }^{39}$ J. H. Park, M. G. Kim, H. M. Jang, S. Ryu, and Y. M. Kim, Appl. Phys. Lett. 84, 1338 (2004).

${ }^{40}$ K. Rode et al., Appl. Phys. Lett. 92, 012509 (2008).

${ }^{41}$ M. Opel, K. W. Nielsen, S. Bauer, S. T. B. Goennenwein, J. C. Cezar, D. Schmeisser, J. Simon, and W. Mader, Eur. Phys. J. B 63, 437 (2008).

${ }^{42}$ T. C. Kaspar, T. Droubay, S. M. Heald, M. H. Engelhard, P. Nachimuthu, and S. A. Chambers, Phys. Rev. B 77, 201303 (2008).

${ }^{43}$ N. H. Hong, J. Sakai, N. Poirot, and V. Brize, Phys. Rev. B 73, 132404 (2006).

${ }^{44}$ M. M. Cruz, R. C. da Silva, N. Franco, and M. Godinho, J. Phys. Condens. Matter 21, 206002 (2009).

${ }^{45}$ N. H. Hong, N. Poirot, and J. Sakai, Phys. Rev. B 77, 033205 (2008).

${ }^{46}$ T. C. Kaspar, T. Droubay, S. M. Heald, P. Nachimuthu, C. M. Wang, V. Shutthanandan, C. A. J. D. R. Gamelin, and S. A. Chambers, New J. Phys. 10, 055010 (2008).

${ }^{47}$ E. C. Lee and K. J. Chang, Phys. Rev. B 69, 085205 (2004).

${ }^{48}$ E. J. Kan, L. F. Yuan, and J. Yang, J. Appl. Phys. 102, 033915 (2007).
${ }^{49}$ S. Lany and A. Zunger, Phys. Rev. B 78, 235104 (2008).

${ }^{50}$ M. H. F. Sluiter, Y. Kawazoe, P. Sharma, A. Inoue, A. R. Raju, C. Rout, and U. V. Waghmare, Phys. Rev. Lett. 94, 187204 (2005).

${ }^{51}$ Q. Wang, Q. Sun, G. Chen, Y. Kawazoe, and P. Jena, Phys. Rev. B 77, 205411 (2008).

${ }^{52}$ J. B. Yi et al., Phys. Rev. Lett. 104, 137201 (2010).

${ }^{53}$ E. Nielsen and R. N. Bhatt, Phys. Rev. B 82, 195117 (2010).

${ }^{54}$ T. Herrmann and W. Nolting, Solid State Commun. 103, 351 (1997).

${ }^{55}$ A. F. Kohan, G. Ceder, D. Morgan, and C. G. V. de Walle, Phys. Rev. B 61, 15019 (2000).

${ }^{56}$ S. B. Zhang, S. H. Wei, and A. Zunger, Phys. Rev. B 63, 075205 (2001).

${ }^{57}$ A. Janotti and C. G. V. de Walle, Appl. Phys. Lett. 87, 122102 (2005).

${ }^{58}$ A. Janotti and C. G. V. de Walle, Phys. Rev. B 76, 165202 (2007).

${ }^{59}$ X. J. Wang, L. S. Vlasenko, S. J. Pearton, W. M. Chen, and I. A. Buyanova, J. Phys. D: Appl. Phys. 42, 175411 (2009).

${ }^{60}$ T. Chanier, I. Opahle, M. Sargolzaei, R. Hayn, and M. Lannoo, Phys. Rev. Lett. 100, 026405 (2008).

${ }^{61}$ Z. Q. Chen, S. J. Wang, M. Maekawa, A. Kawasuso, H. Naramoto, X. L. Yuan, and T. Sekiguchi, Phys. Rev. B 75, 245206 (2007).

${ }^{62}$ D. Galland and A. Hervé, Phys. Lett. A 33, 1 (1970).

${ }^{63}$ L. S. Vlasenko and G. D. Watkins, Phys. Rev. B 71, 125210 (2005).

${ }^{64}$ S. M. Evand, N. C. Giles, L. E. Halliburton, and L. A. Kappers, J. Appl. Phys. 103, 043710 (2008).

${ }^{65}$ C. V. de Walle and J. Neugebauer, J. Appl. Phys. 95, 3851 (2004).

${ }^{66} \mathrm{~J}$. A. Dean, Lange's Handbook of Chemistry, 14th ed. (McGrawHill, New York, 1992).

${ }^{67}$ R. Dovesi et al., CRYSTAL06 User's Manual, University of Torino, Torino (2007).

${ }^{68}$ G. Makov and M. C. Payne, Phys. Rev. B 51, 4014 (1995).

${ }^{69}$ P. A. M. Dirac, Proc. Cambridge Philos. Soc. 26, 376 (1930).

${ }^{70}$ S. H. Vosko, L. Wilk, and M. Nusair, Can. J. Phys. 58, 1200 (1980).

${ }^{71}$ A. D. Becke, Phys. Rev. A 38, 3098 (1988).

${ }^{72}$ C. Lee, W. Yang, and R. G. Parr, Phys. Rev. B 37, 785 (1988).

${ }^{73}$ J. Heyd, G. E. Scuseria, and M. Ernzerhof, J. Chem. Phys. 118, 8207 (2003).

${ }^{74}$ J. Heyd, G. E. Scuseria, and M. Ernzerhof, J. Chem. Phys. 124, 219906 (2006)

${ }^{75}$ F. Oba, A. Togo, I. Tanaka, J. Paier, and G. Kresse, Phys. Rev. B 77, 245202 (2008).

${ }^{76}$ P. Erhart, K. Albe, and A. Klein, Phys. Rev. B 73, 205203 (2006).

${ }^{77}$ R. Vidya, P. Ravindran, H. Fjellvag, B. G. Svensson, E. Monakhov, M. Ganchenkova, and R. M. Nieminen, Phys. Rev. B 83, 045206 (2011).

${ }^{78}$ F. Gallino, G. Paccioni, and C. DiValentin, J. Chem. Phys. 133, 144512 (2010)

${ }^{79}$ J. F. Janak, Phys. Rev. B 18, 7165 (1978).

${ }^{80}$ P. Gopal and N. A. Spaldin, Phys. Rev. B 74, 094418 (2006).

${ }^{81}$ T. Chanier, M. Sargolzaei, I. Opahle, R. Hayn, and K. Koepernik, Phys. Rev. B 73, 134418 (2006).

${ }^{82}$ M. Venkatesan, C. B. Fitzgerald, and J. M. D. Coey, Nature (London) 430, 630 (2004).

${ }^{83}$ J. B. Yi, H. Pan, J. Y. Lin, J. Ding, Y. P. Feng, S. Thongmee, T. Liu, H. Gong, and L. Wang, Adv. Mater. 20, 1170 (2008).

${ }^{84}$ Z. Yan, Y. Ma, D. Wang, J. Wang, Z. Gao, L. Wang, P. Yu, and T. Song, Appl. Phys. Lett. 92, 081911 (2008). 
${ }^{85}$ Q. Xu et al., Appl. Phys. Lett. 92, 082508 (2008).

${ }^{86}$ J. E. Jaffe and A. C. Hess, Phys. Rev. B 48, 7903 (1993).

${ }^{87}$ L. Valenzano, J. F. Torres, K. Doll, F. Pascale, C. M. ZicovichWilson, and R. Dovesi, Z. Phys. Chem. 220, 893 (2006).

${ }^{88}$ M. D. Towler, N. L. Allan, N. M. Harrison, V. R. Saunders, W. C. Mackrodt, and E. Aprà, Phys. Rev. B 50, 5041 (1994).

${ }^{89}$ A. D. Becke, J. Chem. Phys. 98, 5648 (1993).
${ }^{90}$ P. J. Stephens, F. J. Devlin, C. F. Chabalowski, and M. J. Frisch, J. Phys. Chem. 98, 11623 (1994).

${ }^{91}$ A. Kokalj, Comput. Mater. Sci. 28, 155 (2003).

${ }^{92}$ Code available from [http://www.xcrysden.org].

${ }^{93}$ C. L. Bailey, L. Liborio, G. Mallia, S. Tomië, and N. M. Harrison, Phys. Rev. B 81, 205214 (2010).

${ }^{94}$ C. Freysoldt, J. Neugebauer, and C. V. de Walle, Phys. Rev. Lett. 102, 016402 (2009). 